Louisiana State University

LSU Digital Commons

7-1-2004

\title{
Spectroscopic observations and analysis of the peculiar SN 1999aa
}

\author{
G. Garavini \\ Stockholms universitet \\ G. Folatelli \\ Stockholms universitet
}

A. Goobar

Stockholms universitet

S. Nobili

Stockholms universitet

G. Aldering

Lawrence Berkeley National Laboratory

See next page for additional authors

Follow this and additional works at: https://digitalcommons.Isu.edu/physics_astronomy_pubs

\section{Recommended Citation}

Garavini, G., Folatelli, G., Goobar, A., Nobili, S., Aldering, G., Amadon, A., Amanullah, R., Astier, P., Balland, C., Blanc, G., Burns, M., Conley, A., Dahlén, T., Deustua, S., Ellis, R., Fabbro, S., Fan, X., Frye, B., Gates, E., Gibbons, R., Goldhaber, G., Goldman, B., Groom, D., Haissinski, J., Hardin, D., Hook, I., Howell, D., Kasen, D., Kent, S., Kim, A., Knop, R., Lee, B., \& Lidman, C. (2004). Spectroscopic observations and analysis of the peculiar SN 1999aa. Astronomical Journal, 128 (1 1783), 387-404. https://doi.org/10.1086/421747

This Article is brought to you for free and open access by the Department of Physics \& Astronomy at LSU Digital Commons. It has been accepted for inclusion in Faculty Publications by an authorized administrator of LSU Digital Commons. For more information, please contact ir@lsu.edu. 


\section{Authors}

G. Garavini, G. Folatelli, A. Goobar, S. Nobili, G. Aldering, A. Amadon, R. Amanullah, P. Astier, C. Balland, G. Blanc, M. S. Burns, A. Conley, T. Dahlén, S. E. Deustua, R. Ellis, S. Fabbro, X. Fan, B. Frye, E. L. Gates, R. Gibbons, G. Goldhaber, B. Goldman, D. E. Groom, J. Haissinski, D. Hardin, I. M. Hook, D. A. Howell, D. Kasen, S. Kent, A. G. Kim, R. A. Knop, B. C. Lee, and C. Lidman 


\section{Lawrence Berkeley National Laboratory}

Lawrence Berkeley National Laboratory

\section{Title}

Spectroscopic observations and analysis of the peculiar SN 1999aa

Permalink

https://escholarship.org/uc/item/0bh468ra

\section{Authors}

Garavini, G.

Folatelli, G.

Goobar, A.

et al.

\section{Publication Date}

2003-12-10

Peer reviewed 


\title{
SPECTROSCOPIC OBSERVATIONS AND ANALYSIS OF THE PECULIAR SN 1999aa
}

\author{
G. Garavini, ${ }^{1}$ G. Folatelli, ${ }^{1}$ A. Goobar,,${ }^{1}$ S. Nobili, ${ }^{1}$ G. Aldering, ${ }^{2,3}$ A. Amadon, ${ }^{4}$ R. Amanullah, ${ }^{1}$ P. Astier,${ }^{5}$ \\ C. Balland ${ }^{5,6}$ G. Blanc, ${ }^{2,7}$ M. S. Burns,${ }^{8}$ A. Conley, ${ }^{2,3,9}$ T. Dahlén, ${ }^{10,11}$ S. E. Deustua,,${ }^{2,12}$ R. Ellis, ${ }^{13}$ \\ S. Fabbro, ${ }^{14}$ X. Fan,${ }^{15}$ B. Frye, ${ }^{2}$ E. L. Gates, ${ }^{16}$ R. Gibbons, ${ }^{2}$ G. Goldhaber,,${ }^{2,9}$ B. Goldman, ${ }^{17}$ D. E. Groom, ${ }^{2}$ \\ J. Haissinski, ${ }^{18}$ D. Hardin, ${ }^{5}$ I. M. Hook ${ }^{19}$ D. A. Howell, ${ }^{2}$ D. Kasen, ${ }^{2}$ S. Kent, ${ }^{20}$ A. G. Kim, ${ }^{2}$ R. A. Knop ${ }^{21}$ \\ B. C. Lee, ${ }^{2}$ C. Lidman, ${ }^{22}$ J. Mendez, ${ }^{23,24}$ G. J. Miller,${ }^{25,26}$ M. Moniez, ${ }^{18}$ A. Mourão, ${ }^{14}$ H. Newberg,${ }^{27}$ \\ P. E. Nugent, ${ }^{2}$ R. Pain,${ }^{5}$ O. Perdereau,${ }^{18}$ S. Perlmutter, ${ }^{2}$ V. Prasad,${ }^{2}$ R. Quimby, ${ }^{2}$ J. Raux,${ }^{5}$ \\ N. Regnault, ${ }^{2}$ J. Rich, ${ }^{4}$ G. T. Richards, ${ }^{28}$ P. Ruiz-Lapuente, ${ }^{24}$ G. Sainton, ${ }^{5}$ B. E. Schaefer,${ }^{29}$ \\ K. Schahmaneche, ${ }^{5}$ E. Smith, ${ }^{21}$ A. L. Spadafora, ${ }^{2}$ V. Stanishev, ${ }^{1}$ N. A. Walton, ${ }^{30}$ \\ L. WANG, ${ }^{2}$ AND W. M. WOOD-VASEY 2,9 \\ (The Supernova Cosmology Project). \\ Received 2003 December 23; accepted 2004 April 7
}

\begin{abstract}
We present an extensive new time series of spectroscopic data of the peculiar SN 1999aa in NGC 2595. Our data set includes 25 optical spectra between -11 and +58 days with respect to $B$-band maximum light, providing an unusually complete time history. The early spectra resemble those of an SN 1991T-like object but with a relatively strong $\mathrm{Ca} \mathrm{H}$ and $\mathrm{K}$ absorption feature. The first clear sign of $\mathrm{Si}$ II 26355 , characteristic of Type Ia supernovae, is found at day -7 , and its velocity remains constant up to at least the first month after $B$-band maximum light. The transition to normal-looking spectra is found to occur earlier than in SN 1991T, suggesting SN 1999aa as a possible link between SN 1991T-like and Branch-normal supernovae. Comparing the observations with synthetic spectra, doubly ionized $\mathrm{Fe}, \mathrm{Si}$, and $\mathrm{Ni}$ are identified at early epochs. These are characteristic of SN 1991T-like objects. Furthermore, in the day -11 spectrum, evidence is found for an absorption feature that could be identified as high velocity C II $\lambda 6580$ or $\mathrm{H} \alpha$. At the same epoch C III $\lambda 4648.8$ at photospheric velocity is probably responsible for the absorption feature at $4500 \AA$. High-velocity Ca is found around maximum light together with $\mathrm{Si}$ II and $\mathrm{Fe}$ II confined in a narrow velocity window. Implied constraints on supernovae progenitor systems and explosion hydrodynamic models are briefly discussed.
\end{abstract}

Key words: supernovae: general — supernovae: individual (SN 1999aa)

\footnotetext{
${ }^{1}$ Department of Physics, Stockholm University, AlbaNova University Center, S-106 91 Stockholm, Sweden.

${ }^{2}$ Lawrence Berkeley National Laboratory, 1 Cyclotron Road, Berkeley, CA 94720.

3 Visiting Astronomer, Cerro Tololo Inter-American Observatory, National Optical Astronomy Observatory, which is operated by the Association of Universities for Research in Astronomy, (AURA), Inc., under cooperative agreement with the National Science Foundation.

${ }_{5}^{4}$ DAPNIA-SPP, CEA Saclay, F-91191 Gif-sur-Yvette, France.

${ }^{5}$ Laboratoire de Physique Nucléaire et de Hautes Energies, CNRS-IN2P3, University of Paris VI and VII, Paris, France.

${ }^{6}$ Université Paris Sud, IAS-CNRS, bâtiment 121, F-91405 Orsay Cedex, France.

7 Osservatorio Astronomico di Padova, INAF, vicolo dell'Osservatorio 5, I-35122 Padova, Italy.

${ }^{8}$ Colorado College, 14 East Cache La Poudre Street, Colorado Springs, CO 80903

9 Department of Physics, University of California, Berkeley, 94720-7300 CA.

${ }^{10}$ Stockholm Observatory, AlbaNova University Center, S-106 91 Stockholm, Sweden.

${ }^{11}$ Space Telescope Science Institute, 3700 San Martin Drive, Baltimore, MD 21218.

${ }_{12}$ American Astronomical Society, 2000 Florida Avenue, NW, Suite 400, Washington, DC, 20009.

${ }_{13}$ California Institute of Technology, East California Boulevard, Pasadena, CA 91125 .

${ }^{14}$ Centro Multidisplinar de Astrofísica (CENTRA) and Departmento de Física, IST, Universidade Técnico de Lisboa, Avenida Rovisco Pais, P-1049001 Lisboa, Portugal.

${ }^{15}$ Steward Observatory, University of Arizona, 933 North Cherry Avenue, Tucson, AZ 85721.

${ }^{16}$ UCO/Lick Observatory, Univeristy of California, Santa Cruz, CA 95064.

17 Department of Astronomy, New Mexico State University, Department 4500, P.O. Box 30001, Las Cruces, NM 88011.
}

\section{INTRODUCTION}

The observed homogeneity and brightness of Type Ia supernovae (SNe Ia) make them excellent tools for distance estimates over extremely large distances and hence for measurements of cosmological parameters (see, e.g., Perlmutter et al. 1998, 1999; Garnavich et al. 1998; Schmidt et al. 1998;

\footnotetext{
${ }^{18}$ Laboratoire de l'Accélerateur Linéaire, IN2P3-CNRS, Université Paris Sud, B.P. 34, 91898 Orsay Cedex, France.

19 Department of Physics, University of Oxford, Nuclear and Astrophysics Laboratory, Keble Road, Oxford OX1 3RH, UK.

${ }^{20}$ Fermi National Accelerator Laboratory, P.O. Box 500, Batavia, IL 60510.

21 Department of Physics and Astronomy, Vanderbilt University, Nashville, TN 37240 .

${ }^{22}$ European Southern Observatory, Alonso de Cordova 3107, Vitacura, Casilla 19001, Santiago 19, Chile.

23 Isaac Newton Group, Apartado de Correos 321, Santa Cruz de La Palma, E-38780 Tenerife, Spain.

${ }^{24}$ Department of Astronomy, University of Barcelona, Martí i Franques 1, E-08028 Barcelona, Spain.

${ }^{25}$ Department of Astronomy, San Diego State University, 5500 Campanile Drive, San Diego, CA 92182-1221.

${ }^{26}$ Department of Astronomy, University of Illinois, 1002 West Green Street Urbana, IL 61801.

27 Department of Physics, Rensselaer Polytechnic Institute, SC1C25, Troy, NY 12180

${ }^{28}$ Princeton University Observatory, Peyton Hall, Princeton, NJ 08544.

29 Department of Physics and Astronomy, Louisiana State University, Baton Rouge, LA 70803.

${ }^{30}$ Institute of Astronomy, Madingley Road, Cambridge CB3 0HA, UK.
}

1 (V128/203552) 5/11/04 
Riess et al. 1998; Knop et al. 2003; Tonry et al. 2003). However, cosmological results derived from supernovae rely on the evidence that distant explosions are similar to wellstudied nearby ones and that they can be calibrated with the same techniques, e.g., the luminosity-light curve-timescale relation (Phillips 1993). The Supernova Cosmology Project (SCP) coordinated an extensive campaign to study a large number of $z<0.1$ SNe Ia in the spring of 1999 in order to better understand the intrinsic properties of $\mathrm{SNe}$ Ia and thereby improve cosmological measurements using them (Aldering 2000; Nugent et al. 2000). The subject of this work, SN 1999aa, was one of the SCP targets in that campaign.

At this time, several fundamental questions about Type Ia supernova physics remain. The nature of the progenitor system is still poorly constrained, as are the details of the explosion and thus the origin of many of the differences observed among $\mathrm{SNe}$ Ia . Normal SNe Ia (sometimes called Branch-normal; Branch et al. 1983, 1993) present early spectra dominated by intermediate mass elements (IMEs), such as $\mathrm{Mg}, \mathrm{Si}, \mathrm{S}$, and $\mathrm{Ca}$, which are replaced by features due to iron-peak ions (such as $\mathrm{Fe}, \mathrm{Co}$, and $\mathrm{Ni}$ ) as the spectrum evolves with time. However, peculiar events such as SN 1991T, SN 1997br, and SN 2000cx (Filippenko et al. 1992; Jeffery et al. 1992; Phillips et al. 1992; Ruiz-Lapuente et al. 1992) show different characteristics. Their early spectra have weak IME lines and strong doubly ionized iron lines, while spectra after maximum light look almost completely normal. Moreover, their light curves are generally characterized by a slow postmaximum decline rate, and thus, SN 1991T-like supernovae are sometimes called peculiar slow decliners. These characteristics have been regarded as possible signs of different classes of progenitors.

SN 1999aa exhibited spectral characteristics common to both Branch-normal and peculiar slow decliner SNe Ia and thus has been proposed as a key object that may help in understanding the physical origin of the observed diversity (Branch 2001a, 2001b; Li et al. 2001b).

Hydrodynamic models predict atmospheric compositions of supernovae in good agreement with what is found in observed spectra. None of the currently available models, though, produce an exhaustive description of the whole spectral time evolution, and none are able to reproduce the complete range of observed differences among SNe Ia. For extensive reviews of the theoretical models and observations see Filippenko (1997), Hillebrandt \& Niemeyer (2000), Leibundgut (2000), Livio (2001), and Branch et al. (2003a).

The most widely accepted model for SNe Ia involves the thermonuclear disruption of a $\mathrm{C}+\mathrm{O}$ white dwarf star (WD) accreting material from a companion star (Whelan \& Iben 1973; Nomoto 1982; Iben \& Tutukov 1984; Paczynski 1985). From an observational point of view this conclusion is supported by the amount of energy released, the absence of hydrogen lines (but see Marietta et al. 2000), and the occurrence of SNe Ia in elliptical galaxies exclusive of other types.

A more controversial issue is whether SNe Ia are the result of explosions at Chandrasekhar mass (Arnett 1969; Hansen \& Wheeler 1969; Nomoto et al. 1976; Khokhlov 1991; Woosley \& Weaver 1994) or at sub-Chandrasekhar mass (Livne 1990; Livne \& Glasner 1991; Woosley \& Weaver 1994). In the former, thermonuclear burning of carbon occurs in proximity to the center of the star and the burning front proceeds outward. In the latter, helium accreting in the external layer of the supernova ignites. A detonation then propagates outward through the He layer and another inward compressing the $\mathrm{C}+\mathrm{O}$ nucleus that ignites off-center.
While Chandrasekhar mass models have been successful in reproducing many of the observed characteristics of Branchnormal and SN 1991T-like supernovae (Nugent et al. 1997; Fisher et al. 1999), sub-Chandrasekhar models are in good agreement with fainter explosions such as SN 1991bg-like objects (Nugent et al. 1997).

Premaximum spectra can discriminate among these two scenarios since sub-Chandrasekhar models have external layers dominated by $\mathrm{He}$ and $\mathrm{Ni}$ and do not leave any unburned carbon or produce IMEs at expansion velocities above $14000 \mathrm{~km} \mathrm{~s}^{-1}$. The identification of $\mathrm{C}$ or strong $\mathrm{Ca}$ or $\mathrm{Si}$ lines in premaximum spectra would then rule out this possibility.

Most of the supernovae observed seem to show characteristics in agreement with carbon ignition occurring at the center of the WD. However, this condition can be the result of both a single degenerate $\mathrm{C}+\mathrm{O}$ WD accreting hydrogen from a companion or a merging double degenerate $\mathrm{C}+\mathrm{O} \mathrm{WD}$, as is sometimes suggested for SN 1991T-like supernovae (Fisher et al. 1999).

The detection of narrow hydrogen lines in supernova spectra (Hamuy et al. 2003; see also a discussion in Livio \& Riess 2003) would favor the single degenerate scenario but a nondetection cannot rule out these models. A substantial amount of hydrogen can be removed from the companion star and get mixed into the exploding WD (Marietta et al. 2000). In this case the detection of $\mathrm{H}$ lines is expected even in low-resolution spectra (Lentz et al. 2002) and would then exclude a double degenerate progenitor system.

While single degenerate models are currently considered the most promising, other questions remain open. The hydrodynamics of the explosion and the details of the flame propagation pose many numerical and conceptual problems currently under investigation, e.g., computational resolution in threedimensional simulations, and details of flame instabilities, for extensive discussion see, e.g., Hillebrandt \& Niemeyer (2000) and Blinnikov \& Sorokina (2004). Several models have been proposed to describe the explosion mechanism. Next, we highlight the possible spectroscopic observables that could help in constraining the parameters of the models.

Pure one-dimensional deflagration models (such as W7; Nomoto et al. 1984) have carbon present down to $v>$ $14,900 \mathrm{~km} \mathrm{~s}^{-1}$, while one-dimensional delayed-detonation (DD) models (Khokhlov 1991; Yamaoka et al. 1992; Woosley \& Weaver 1994) burn $\mathrm{C}$ almost completely up to $v \sim$ $30,000 \mathrm{~km} \mathrm{~s}^{-1}$. Thus, the identification of carbon lines at low velocity would disfavor published DD models. Delayeddetonation models can also produce an unusually high Doppler blueshift of IMEs by tuning the deflagration to detonation transition density (Lentz et al. 2001). Thus, IME lines confined at higher than normal velocities could be more naturally described by DD models. Lines of stable $\mathrm{Fe}$ and $\mathrm{Ni}$ at high velocity in the spectra prior to maximum are also consistent with the prediction of a deflagration-to-detonation transition (Iwamoto et al. 1999).

The complexity of explosion models has advanced to the level where deflagration in three-dimensions can be explored (Khokhlov 2000; Gamezo et al. 2003). These results motivated a few attempts to investigate the spectral outcome of these models by means of parametrized synthetic spectra codes (Thomas et al. 2002; Baron et al. 2003; Kasen et al. 2003). In three-dimensional deflagration, because of the highly convoluted turbulent flame propagation, heavy mixing of freshly synthesized and unburned material can occur (Gamezo et al. 2003). Evidence of $\mathrm{C}$ and $\mathrm{O}$ lines at low velocities, as well 


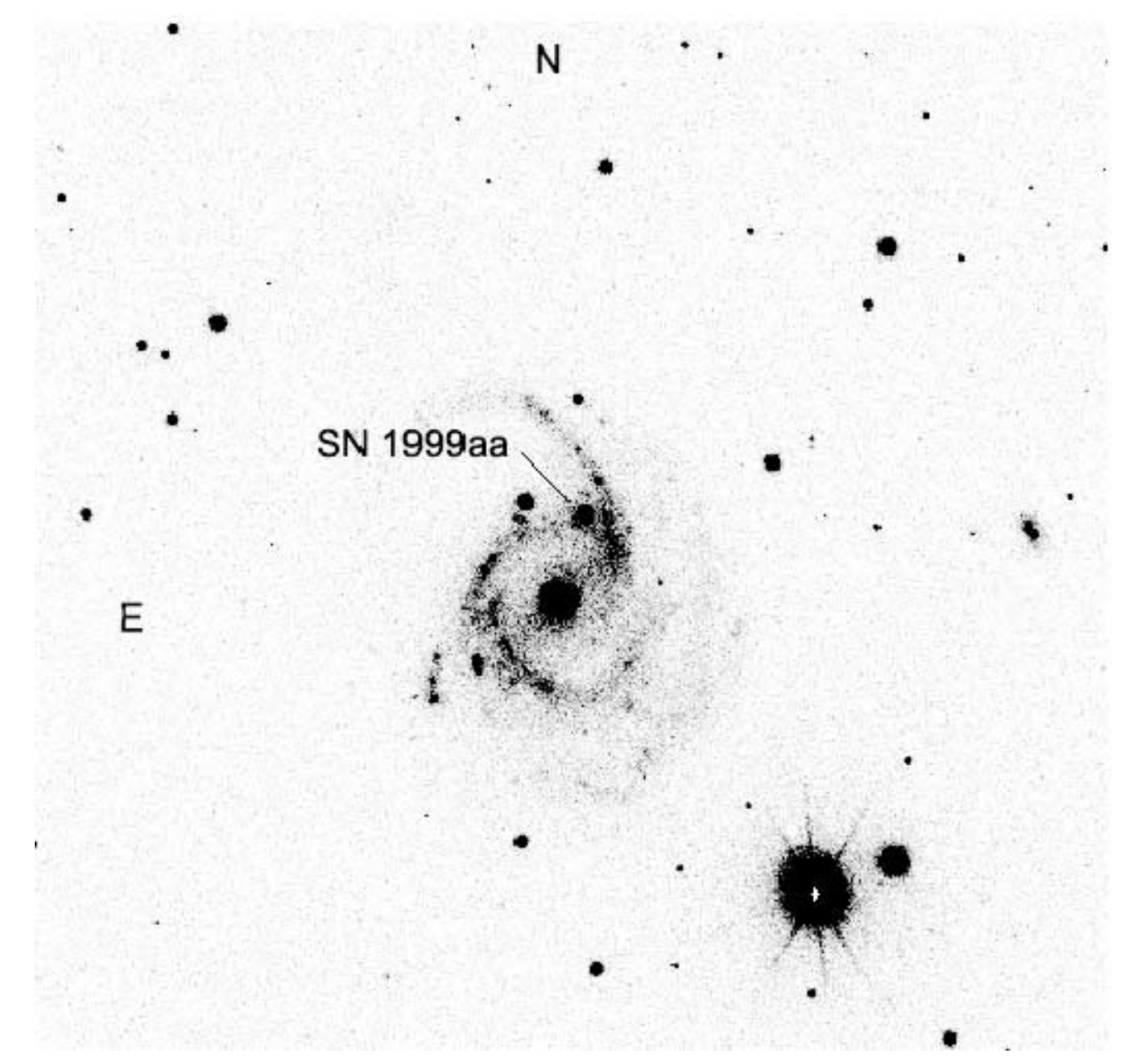

Fig. 1. - SN 1999aa in its host galaxy NGC 2595 , R.A. $=8^{\mathrm{h}} 27^{\mathrm{m}} 42^{\circ} .03$, decl. $=+21^{\circ} 29^{\prime} 14^{\prime \prime} .8(\mathrm{~J} 2000.0)$. The $B$-band image was obtained at NOT on 1999 February 13 UT with SN 1999aa indicated. The field is 6.5 across. North is up, and east is to the left.

as clumps of burned material (e.g., IME or Fe) in the external layers (high velocity), would confirm the plausibility of, and the need for, such a degree of complexity. The same mechanism that mixes carbon and oxygen with IME in threedimensional calculations could probably also work for hydrogen.

Polarization measurements in SNe Ia spectra (Wang et al. 1996, 2003; Howell et al. 2001; Kasen et al. 2003) have strengthened the conviction that some degree of asymmetry can be found in supernova atmospheres. In particular, the high-velocity (HV) component of the Ca II IR triplet found in some supernovae (e.g., SN 2000cx, SN 2001el) require threedimensional simulations to be fully reproduced (Thomas et al. 2003; Kasen et al. 2003).

To explore the results of three-dimensional explosion models, full three-dimensional radiative transfer calculations of supernova spectra are required. However, these are not yet available and one-dimensional parametrized radiative transfer calculations are still in their infancy. Thus, direct analysis by means of parametrized codes - both in one and three dimensions - remains the fastest and most versatile way of testing model predictions and guiding new developments.

Here we present a new, comprehensive spectroscopic time series for SN 1999aa. We use the direct analysis code SYNOW (Jeffery \& Branch 1990; Fisher et al. 1997, 1999) as a tool to describe the data and to identify potentially interesting features. This, together with the measurements of the velocities inferred from the minima of the spectral features, is used to investigate the structure of the expanding atmosphere. In particular, we have looked for evidence of carbon, oxygen, and hydrogen lines in early spectra, as well as the velocity ranges of iron, nickel, and intermediate mass element lines, in order to try to answer some of the open questions outlined above.

This paper is organized as follows. The spectroscopic data of SN 1999aa and a short description of the data reduction scheme are presented in $\S 2$. The analysis methodology is introduced in $\S 3$. In $\S 4$ our SN 1999aa spectra are compared with those of spectroscopically peculiar and normal supernovae taken from the literature. The synthetic spectra for days $-11,-1,+5,+14$, and +40 , produced with the highly parametrized SYNOW code, are discussed in the same section that outlines the spectral peculiarities of this object. Velocities inferred from several spectral features are analyzed in $\S 5$. A discussion about possible implications for supernova models is presented in $\S 6$ and our conclusions are given in $\S 7$.

\section{DATA SET AND REDUCTION PROCEDURE}

SN 1999aa (R.A. $=8^{\mathrm{h}} 27^{\mathrm{m}} 42^{\mathrm{s}} .03$, decl. $=+21^{\circ} 29^{\prime} 14^{\prime \prime} .8$, J2000.0) in NGC 2595 was discovered independently by Armstrong \& Schwartz (1999), Qiao et al. (1999), and Nakano et al. (1999) on 1999 February 11. Filippenko et al. (1999) noted that it was an SN 1991T-like SNe Ia based on a spectrum taken the following night. Figure 1 shows the position of 
TABLE 1

Data Set Specifications

\begin{tabular}{|c|c|c|c|c|c|c|c|}
\hline $\begin{array}{c}\mathrm{JD} \\
(-2,400,000)\end{array}$ & $\begin{array}{c}\text { Epoch }^{\mathrm{a}} \\
\left(\operatorname{Ref} B_{\max }\right)\end{array}$ & Telescope & Instrument & $\begin{array}{c}\lambda \text { Range }^{\mathrm{a}, \mathrm{b}} \\
(\AA)\end{array}$ & $\begin{array}{c}\langle\Delta \lambda\rangle^{\mathrm{a}, \mathrm{c}} \\
(\AA)\end{array}$ & $\langle\mathrm{S} / \mathrm{N}\rangle^{\mathrm{d}}$ & Comments \\
\hline $51223.38 \ldots \ldots \ldots \ldots \ldots$ & -11 & NOT $2.6 \mathrm{~m}$ & ALFOSC & $3503-9295$ & 6.1 & 52 & $5998^{\mathrm{e}}$ \\
\hline $51227.84 \ldots \ldots \ldots \ldots \ldots$ & -7 & APO $3.5 \mathrm{~m}$ & DIS & $3686-10268$ & 6.8 & 75 & $5490^{\mathrm{e}}$ \\
\hline $51231.35 \ldots \ldots \ldots \ldots \ldots$ & -3 & NOT $2.6 \mathrm{~m}$ & ALFOSC & $3508-9692$ & 6.1 & 41 & $6097^{\mathrm{e}}$ \\
\hline $51234.71 \ldots \ldots \ldots \ldots \ldots$ & -1 & Lick 3 m & KAST & $3478-9975$ & 3.1 & 43 & $5430^{\mathrm{e}}$ \\
\hline $51239.68 \ldots \ldots \ldots \ldots \ldots$ & +5 & MDM $2.4 \mathrm{~m}$ & MARK III & $3872-8807$ & 5.3 & 45 & $\mathrm{f}$ \\
\hline $51239.68 \ldots \ldots \ldots \ldots . . . . . .$. & +6 & MDM $2.4 \mathrm{~m}$ & MARK III & $3871-8808$ & 5.3 & 61 & $\mathrm{f}$ \\
\hline $51247.63 \ldots \ldots \ldots \ldots \ldots$ & +14 & APO $3.5 \mathrm{~m}$ & DIS & $3628-10333$ & 6.8 & 86 & $5887^{\mathrm{e}}$ \\
\hline $51253.37 \ldots \ldots \ldots \ldots \ldots$ & +19 & NOT $2.6 \mathrm{~m}$ & ALFOSC & $3375-9994$ & 6.0 & 63 & $6057^{e}$ \\
\hline $51258.51 \ldots \ldots \ldots \ldots \ldots$ & +25 & CTIO 4 m & RCSP & $3320-9208$ & 2.0 & 40 & $\mathrm{~g}$ \\
\hline $51261.54 \ldots \ldots \ldots \ldots \ldots$ & +28 & CTIO $4 \mathrm{~m}$ & RCSP & $3374-9232$ & 2.1 & 42 & $\mathrm{~g}$ \\
\hline $51266.53 \ldots \ldots \ldots \ldots \ldots$ & +33 & CTIO 4 m & RCSP & $3263-9199$ & 2.1 & 42 & g \\
\hline $51273.62 \ldots \ldots \ldots \ldots \ldots$ & +40 & APO $3.5 \mathrm{~m}$ & DIS & $3641-10115$ & 6.7 & $425877^{\mathrm{e}}$ & \\
\hline $51282.67 \ldots \ldots \ldots \ldots \ldots$ & +47 & Lick 3 m & KAST & $3512-9940$ & 3.1 & 18 & $5462^{\mathrm{e}}$ \\
\hline $51286.63 \ldots \ldots \ldots \ldots \ldots$ & +51 & APO $3.5 \mathrm{~m}$ & DIS & $3621-10166$ & 6.7 & 68 & $5874^{\mathrm{e}}$ \\
\hline $51293.71 \ldots \ldots \ldots \ldots \ldots$ & +58 & Lick 3 m & KAST & $3705-7963$ & 2.1 & 11 & $5390^{\mathrm{e}}$ \\
\hline
\end{tabular}

Note.-(APO): Apache Point Observatory; (CTIO): Cerro Tololo Inter-American Observatory; (Lick): Lick Observatory; (MDM): MDM Observatory; (NOT): Nordic Optical Telescope.

${ }^{\mathrm{a}}$ In rest frame.

${ }^{b}$ Where $\mathrm{S} / \mathrm{N} \geq 5$.

c Average wavelength bin size.

d Average signal-to-noise ratio per wavelength bin.

e Beginning of red channel in angstroms.

f Possible second-order contamination above $7500 \AA$.

g Negligible second-order contamination.

SN 1999aa in its host galaxy NGC 2595, at redshift $z=$ 0.0146 . NGC 2595 is an SBc galaxy with a compact blue core and blue spiral arms. The recession velocity of the host galaxy was determined from narrow $\mathrm{H} \alpha$ and [N $\mathrm{NI}$ ] emission (Armstrong \& Schwartz 1999). According to Schlegel et al. (1998) the Galactic reddening in the direction of SN 1999aa is $E(B-V)=0.04 \mathrm{mag}$. Based on the $\mathrm{SN}$ colors, Krisciunas et al. (2000) concluded that host galaxy reddening is negligible. They also estimated the light curve decline rate $\Delta m_{15}=$ $0.746 \pm 0.024$ from a fourth-order polynomial fit to the light curve. Jha (2002) reported $\Delta m_{15}=0.85 \pm 0.08$ using a different photometry data set.

The SCP follow-up campaign of this supernova involved five different instruments and resulted in 25 optical spectra ranging between 11 days before and 58 days after maximum light (all epochs in this work are given with respect to the $B$-band maximum). In most cases the spectra were acquired using different instrumental settings for the blue and the red parts of the spectrum in order to avoid possible second-order contamination. Whenever both blue and red spectra were taken at the same epoch, we present the combined spectrum. The observations were performed aligning the slit along the parallactic angle in order to minimize light loss from differential atmospheric refraction. Specifications of the data set are provided in Table 1, and the spectral time sequence is shown in Figure 2.

All the raw data were analyzed with a common reduction scheme using standard IRAF routines. The two-dimensional spectra were bias-subtracted and flat-fielded using calibration images taken with the same instrument settings as the $\mathrm{SN}$ spectra and during the same night of observation. In most cases, the observations were split into multiple exposures in order to allow elimination of cosmic rays from the final spectrum. Background subtraction was performed on the resulting spectra using a fitted model of the underlying sky and host

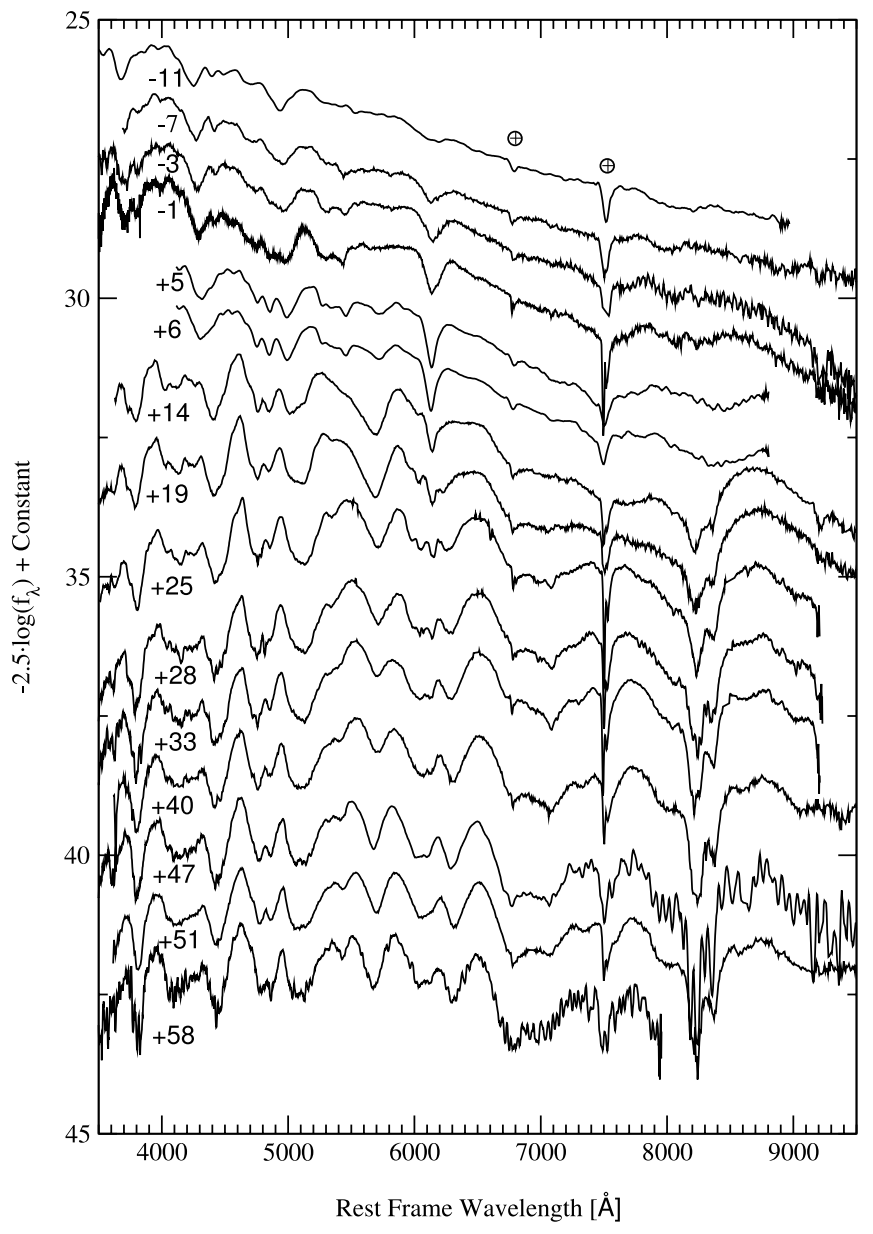

FIG. 2. - SN 1999aa spectral time sequence. The spectra are labeled in days relative to $B$-band maximum light. The circled plus sign marks telluric atmospheric absorptions. 
galaxy signal. The supernova spectrum was extracted using the variance weighted optimal aperture extraction method (Horne 1986). Several arc lamp exposures, generally taken off supernova position, were used for wavelength calibration each night. The accuracy of the calibration was checked against sky lines and no significant deviations were found. Atmospheric extinction corrections were applied using tabulated average extinction curves provided by the observatories. Spectrophotometric standard stars (Oke 1990; Massey \& Gronwall 1990; Turnshek et al. 1990; Hamuy et al. 1992, 1994; Bohlin et al. 1995, 2001; Stone 1996) were observed during each night and their latest calibrations ${ }^{31}$ were used to flux calibrate the SN spectra. A comparison between observations of different standard stars during the same night was used to check for possible systematic errors. A correction for Galactic extinction was performed using the standard procedure in Cardelli et al. (1989) assuming $R_{V}=3.1$. Finally, residual host-galaxy contamination was checked statistically. The spectrum of the host galaxy at the position of the supernova, multiplied by an ar-

Q2 bitrary scale factor, was subtracted from the observed data and compared to a supernova spectral template. The value of the scale factor was determined by minimizing the $\chi^{2}$ between the model (the supernova spectral template) and the data (supernova data plus rescaled host galaxy data). The host galaxy contamination was found to be negligible at all studied epochs. Thus, no (additional) galaxy spectrum was subtracted. Telluric absorptions and residual fringing patterns were left uncorrected because they do not affect our present analysis. For a complete description of the data reduction methodology see Folatelli (2004).

\section{SPECTRAL ANALYSIS METHODOLOGY}

Since we will use SYNOW to produce synthetic spectra and to investigate line identifications and velocity ranges of ions in SN 1999aa, here we briefly review its underlying precepts and parameters. SYNOW generates spectra using a simple conceptual model of a homologously expanding supernova envelope. This model consists of a continuum-emitting, sharply defined photosphere surrounded by an extended line-forming, pure scattering atmosphere. Line transfer is treated using the Sobolev method (Sobolev 1960; Castor 1970; Jeffery \& Branch 1990). Thus, line opacity is parametrized in terms of Sobolev optical depth. The choice of ions used in the calculation is guided by experience and the $\mathrm{SN}$ ion signature atlas of Hatano et al. (1999b). For each ion introduced, the Sobolev optical depth as a function of radius for a "reference line" (usually a strong optical line) is specified. Optical depths in other lines of the ion are set assuming Boltzmann excitation of the levels at temperature $T_{\text {exc }}$.

The parameters $v_{\text {phot }}$ and $T_{\mathrm{bb}}$ set the velocity and blackbody continuum temperature of the photosphere, respectively. For each ion, the optical depth $\tau$ and the specified minimum ejection velocity $v_{\min }$ is given. The optical depth scales exponentially with velocity according to $e$-folding velocity $v_{e}$, up to a maximum velocity given by $v_{\max }$. If $v_{\min }>v_{\text {phot }}$ for an ion, we refer to the ion as "detached."

The blackbody assumption is a basic simplification of the Q3 processes that contribute to the continuum emission; therefore, $T_{\mathrm{bb}}$ cannot be regarded as physical information. Thus, SYNOW produces only a rough indication of the continuum level, which can disagree with the observed spectrum. We

\footnotetext{
31 We have corrected the Hamuy et al. (1992, 1994) spectra for telluric atmospheric features.
}

handled this by bringing the blue portions of the observed and modeled spectra into agreement. The continuum mismatches that then occur at longer wavelengths have not adversely affected our study of the few spectral features present in the red.

Direct analysis results, such as those from SYNOW, yield meaningful constraints for hydrodynamic explosion modelers. Furthermore, direct analysis often reveals the presence of lines that otherwise go undetected without some treatment of the line blending that characterizes supernova spectra.

\section{SPECTRAL DATA AND MODELING}

Prior to $B$-band maximum light, normal SNe Ia have spectra dominated by intermediate mass elements such as Si II, S II, Mg II, Ca II, and O I (Branch et al. 1983, 1993). With time, the absorption features from these elements become weaker and increasingly contaminated by iron-peak element lines. The substitution is usually complete around 30 days after maximum, when the photosphere of the supernova starts receding into the iron-peak core (i.e., the innermost part of the ejecta, where mainly iron-peak elements are present). Objects such as SN 1991T, SN 1997br, and SN 2000cx represent a deviation from the impressive homogeneity of the spectral and photometric characteristics of SN explosions (Fisher et al. 1999; Li et al. 1999, 2001a). This has raised the question whether they could be explained as a different physical phenomenon or as extreme cases of the same process. Ever since the first spectra of SN 1999aa started circulating in the supernova community, several authors have suggested that this object could be helpful in addressing this issue (Branch 2001b; Li et al. 2001b). This work should be viewed in that context.

In order to understand and interpret the differences between SN 1999aa and other normal and peculiar supernovae we now analyze the spectral time evolution through spectral comparison and spectral modeling. We have selected five epochs, approximately 1 week apart from each other, to describe the spectral time evolution in all interesting phases. These are -11 , $-1,+5,+14$, and +40 days with respect to $B$-band maximum.

\subsection{Day 11 Before Maximum}

Our spectrum of SN 1999aa at 11 days prior to $B$-band maximum light is shown in Figure 3. The spectra of SN 1991T, SN 1990N, and SN 1994D are also shown for comparison. The identification of the lines labeled here and in the following graphs follow those in Li et al. (1999, 2001a), Fisher et al. (1999), Patat et al. (1996), Mazzali et al. (1995), Kirshner et al. (1993), and Jeffery et al. (1992). The spectra of SN 1999aa and SN $1991 \mathrm{~T}$ are very different from those of normal supernovae SN 1990N and SN 1994D around 11 days prior to the $B$-band maximum light. Instead of the typical Si II, S II, and Mg II lines, early spectra of these peculiar SNe Ia are dominated by two deep absorptions due to Fe III $\lambda \lambda 4404$ and 5129. Si III $\lambda 4560$ is responsible for the smooth absorption on the red side of the Fe III $\lambda 4404$ line. Possibly weaker Ni III lines contribute near 4700 and $5300 \AA$, (Jeffery et al. 1992; Ruiz-Lapuente et al. 1992). What mainly distinguishes SN 1999aa from SN 1991T is the presence in the former of the trough around $3800 \AA$, most probably due to $\mathrm{Ca}$ II $\mathrm{H}$ and $\mathrm{K}$. This absorption is weak compared to normal SNe Ia. The weak and broad lines around $6150 \AA$ could be due to $\mathrm{Si}$ II $\lambda 6355$ with some contamination from C II $\lambda 6580$.

\subsubsection{Synthetic Spectrum}

In Figure 4 we show the results for the best-match synthetic spectrum computed using the parameters in Table 2. The 


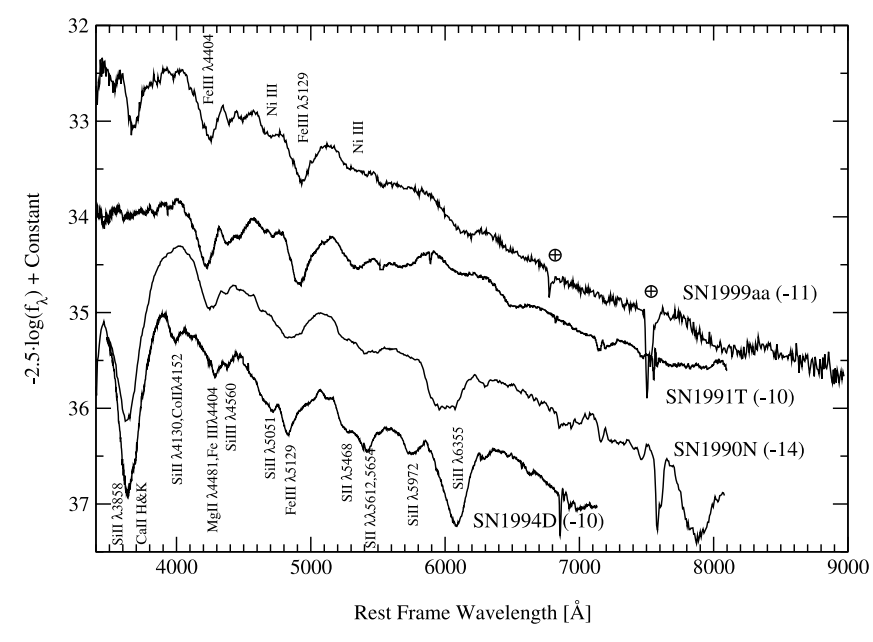

FIG. 3.-Day - 11 spectrum of SN 1999aa together with those of SN 1991T and normal SN 1990N and SN 1994D from Filippenko et al. (1992), Leibundgut et al. (1991), and Patat et al. 1996. Each spectrum is labeled with the phase (days since $B$ maximum). Line identification is explained in the text. The circled plus sign marks atmospheric absorptions.

continuum blackbody temperature is set to $13,700 \mathrm{~K}$, and the photosphere is placed at $11,000 \mathrm{~km} \mathrm{~s}^{-1}$. The dominant and characteristic features at this epoch are due to Fe III lines. While some authors identify the small absorption line at $4000 \AA$ only as $\mathrm{Si}$ II $\lambda 4130$, in our synthetic spectrum we find that $\mathrm{Si}$ II alone cannot reproduce the feature completely because of its small optical depth. If we add a contribution from Co II (as in Hatano et al. 1999a, for example) we are able to reproduce the observed spectrum. Note that to improve the match Co II needs to be detached above the photosphere. Si III appears confined below $16 \times 10^{3} \mathrm{~km} \mathrm{~s}^{-1}$ (similarly to Si II) and is responsible for the features at 4400 and $5500 \AA$, as originally identified by Jeffery et al. (1992) for SN 1991T and SN 1990 N.

The only way we could account for the complete structure of the $\mathrm{Ca}$ II $\mathrm{H}$ and $\mathrm{K}$ features and for its velocity distribution (blue edge at $40,000 \mathrm{~km} \mathrm{~s}^{-1}$ ) was to introduce a HV component (with higher $v_{e}$ ) in addition to the one at $v_{\max }=19500 \mathrm{~km} \mathrm{~s}^{-1}$. This allows us to "fill-up" the whole spectral profile at this epoch, and at day -1 it will produce the observed blue absorption feature of the $\mathrm{Ca}$ II IR triplet, as noticed already in Hatano et al. (1999a) for SN 1994D and analyzed in Kasen et al. (2003) for SN 2001el and in Thomas et al. (2003) for SN 2000cx. The region around $4600 \AA$ and the broad absorption at $5300 \AA$ can be matched with detached Ni III.

We have focused on the wavelength regions around 6150 and $4500 \AA$ for particular study, since these have been discussed for other SNe Ia in the literature. Si II 6150 has a very broad profile. As we show in $\S 5$ the velocity fitted for $\mathrm{Si}$ II 6355 at this epoch is the lowest among all known supernovae $\left(v \sim 8000 \mathrm{~km} \mathrm{~s}^{-1}\right)$. This suggests that probably another ion is partially or totally responsible for this absorption feature. In Figure 3, a similar absorption feature is visible for the spectrum at -10 days of SN 1991T. Fisher et al. (1999) discussed the possible presence of HV C in early spectra of SN 1991T based on the line at $6150 \AA$, usually attributed to Si II 6355 . The same possibility has also been proposed for SN 1990N in Fisher et al. (1997) and Mazzali (2001), for SN 1994D in Hatano et al. (1999a), and recently for SN 1998aq in Branch et al. (2003b).

Guided by the low-velocity value of $\mathrm{Si}$ II 6355 and the similarity with SN 1991T we also introduced an HV C II $\left(v_{\min }=19 \times 10^{3} \mathrm{~km} \mathrm{~s}^{-1}\right)$ in order to try to improve the description of the line profile at $6150 \AA$ Å. Figure 5 shows different SYNOW spectra compared with the data in the 6150 A region. The small notch around $6200 \AA$ is an artifact of the spectrum extraction and does not have to be considered a supernova feature. The error spectrum on the wavelength region $6195-$ $6210 \AA$ is $30 \%$ larger than that of neighboring regions, thus, the small notch does not have large statistical weight for any quantitative measurements. The continuum level of the models has been shifted to match the data in this region. The model at the top has no $\mathrm{C}$ and includes only $\mathrm{Si}$ II. The absence of a $\mathrm{C}$ II component, i.e., considering only Si II, places the minimum of the feature to the blue side of the absorption at $6150 \AA$ and does not completely reproduce the line profile. The parameter $v_{\min }$ is set equal to the photospheric velocity and thus has been determined by looking at all the lines together. The dashed line indicates the synthetic spectrum with the maximum velocity of Si II set to a value $\left(v_{\max }=30 \times 10^{3} \mathrm{~km} \mathrm{~s}^{-1}\right)$ higher than in the later epochs, but which reproduces the line profile more accurately. The model at the bottom of Figure 5 includes both carbon and silicon. Because of the introduction of $\mathrm{C}$ II, we would expect a small absorption (C II 27234) around $6800 \AA$ (close to a telluric line), which is not convincingly visible. It is not fully evident which of the two models proposed matches the data better. The result of a $\chi^{2}$ analysis shows that they are equally plausible. However, the introduction of $\mathrm{C}_{\text {II }}$ would solve the puzzle of the low-velocity value measured for $\mathrm{Si}$ II 26355 . Note that later on (at day -7, Fig. 2) the shape of the feature at $6150 \AA$ changes with the appearance of the first strong Si II $\lambda 6355$ line.

In our spectrum of SN 1999aa we find that the absorption to the red of Si III $\lambda 4568$ can be well matched by C III $\lambda 4648.8$ Q4 confined to low velocities. Its contribution is shown in Figure 6, in which synthetic spectra with and without a $\mathrm{C}$ III component are compared with the observed spectrum. This absorption is unusual for $\mathrm{SNe}$ Ia, and its identification is still unclear. Threedimensional models indicate that unburned doubly ionized $\mathrm{C}$ can, in principle, be visible in the hot interior of the supernova atmosphere (Gamezo et al. 2003). Later in the spectral evolution, the identification of $\mathrm{C}$ could be difficult because of

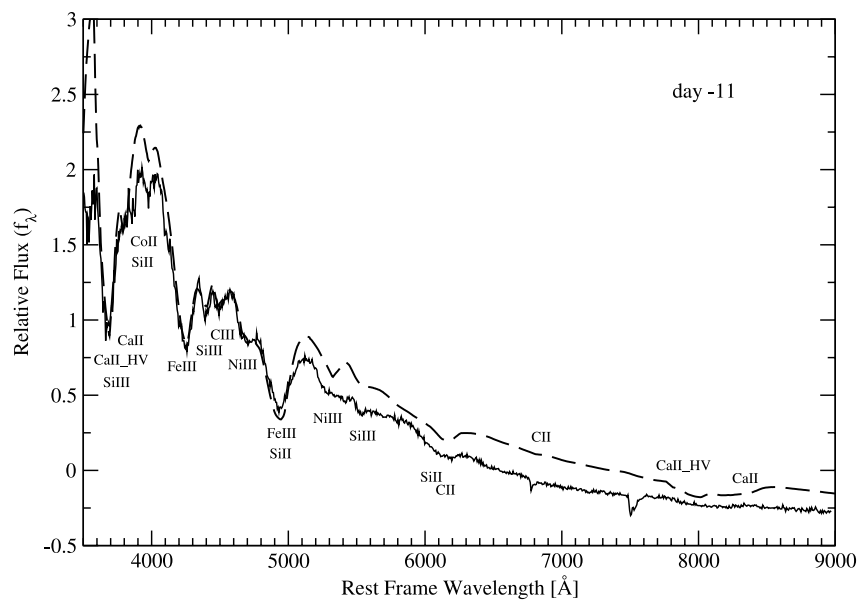

FIG. 4.- Synthetic spectrum compared with SN 1999aa spectrum for day -11 . SYNOW parameters are presented in Table 2. The regions around 6150 and $4500 \AA$ are enlarged in Figs. 5 and 6 to highlight the possible contributions of $\mathrm{C}_{\text {II }}$ and $\mathrm{C}$ III. The mismatch of the continuum level above $5000 \AA$ has to be considered a limitation of the underlying blackbody assumption, but it does not affect the line identification. Ions responsible for features in the synthetic spectrum are labeled. 
TABLE 2

SYNOW PARAMETERS FOR DAY -11

\begin{tabular}{|c|c|c|c|c|c|}
\hline Ion & $\tau$ & $\begin{array}{c}v_{\min } \\
\left(10^{3} \mathrm{~km} \mathrm{~s}^{-1}\right)\end{array}$ & $\begin{array}{c}v_{\max } \\
\left(10^{3} \mathrm{~km} \mathrm{~s}^{-1}\right)\end{array}$ & $\begin{array}{c}T_{\mathrm{exc}} \\
\left(10^{3} \mathrm{~K}\right)\end{array}$ & $\begin{array}{c}v_{e} \\
\left(10^{3} \mathrm{~km} \mathrm{~s}^{-1}\right)\end{array}$ \\
\hline $\mathrm{Ca}$ II................ & 1.15 & $\ldots$ & 19.5 & 15 & 5 \\
\hline $\mathrm{Ca}$ II $(\mathrm{HV})^{\mathrm{a}} \ldots .$. & 1.45 & 19.5 & 30 & 15 & 10 \\
\hline Si II ................. & 0.1 & $\ldots$ & 15 & 15 & 5 \\
\hline Si III ..................... & 0.3 & $\ldots$ & 16 & 15 & 5 \\
\hline Co II................. & 0.25 & 14 & 30 & 10 & 5 \\
\hline Fe III ............... & 0.63 & $\ldots$ & 30 & 10 & 5 \\
\hline Ni III .............. & 2 & 14 & 30 & 10 & 5 \\
\hline $\mathrm{C}$ II................ & 0.01 & 19 & 30 & 15 & 5 \\
\hline $\mathrm{C}$ III................. & 0.5 & - & 13.5 & 15 & 5 \\
\hline
\end{tabular}

Note.-The fit is shown in Fig. 4 ; $v_{\text {phot }}=11000 \mathrm{~km} \mathrm{~s}^{-1}, T_{\mathrm{bb}}=13700 \mathrm{~K}$.

a (HV): High-velocity component.

increased blending of spectral lines. Further, we note that this absorption feature is no longer visible in our spectrum at day -7 (see Fig. 2).

As an alternative to $\mathrm{C}$ III and $\mathrm{C}$ II, $\mathrm{H}$ could be used to match the absorption at 4500 and $6150 \AA$ (Lentz et al. 2002). We discuss these alternative explanations in $\S 6.3$.

\subsection{Day 1 before Maximum}

The spectrum of SN 1999aa around maximum light is shown in Figure 7, together with those of SN 1991T, SN 1981B, and SN 1994D. SN 1999aa resembles that of normal SNe Ia, while SN $1991 \mathrm{~T}$ is still dominated by Fe III lines with very weak $\mathrm{Si}$ II and $\mathrm{Ca} \mathrm{H}$ and $\mathrm{K}$ lines at this epoch. The $\mathrm{Ca}$ II $\mathrm{H}$ and K region of SN 1999aa shows a characteristic split in which the red component is gaining strength. S II appears at this epoch showing the typical $\mathrm{W}$-shaped feature at $\lambda \lambda 5454$, 5606 that SN 1991T-like SNe Ia do not usually show. Si II $\lambda 6355$ is visible but fainter and redder than in normal $\mathrm{SNe}$ Ia. If the evolution from $\mathrm{C}$ II to $\mathrm{Si}$ II proposed by Fisher et al. (1999) is confirmed, SN 1991T at this epoch shows a

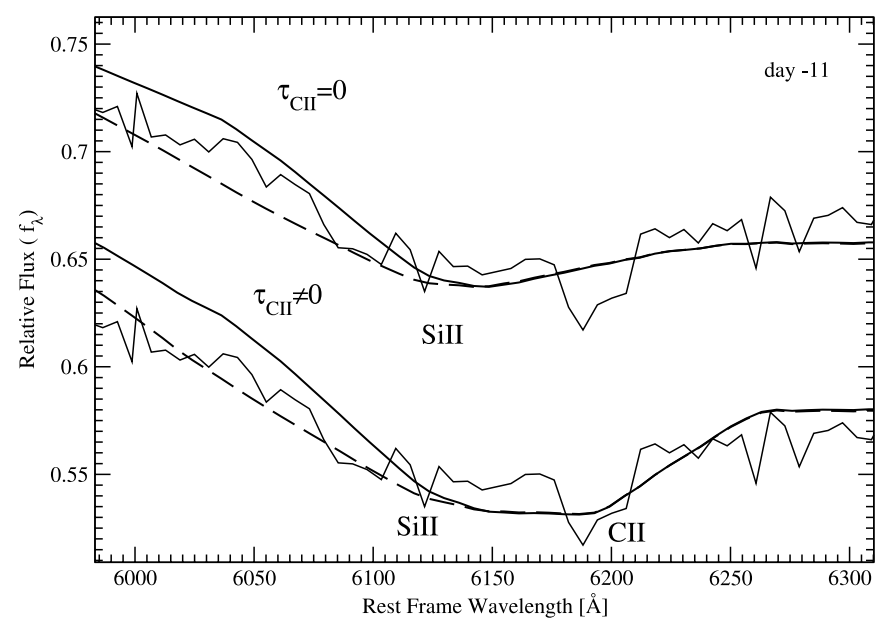

FIG. 5.-Synthetic spectra compared with SN 1999aa spectrum for day -11 around 6150 A. Top model: Solid lines, $\tau_{\mathrm{C} \text { II }}=0$ and data; dashed lines, $\tau_{\mathrm{C} \text { II }}=0$ and Si II with $v_{\max }=30 \times 10^{3} \mathrm{~km} \mathrm{~s}^{-1}$. Bottom model: Solid lines, $\tau_{\mathrm{C} \text { II }} \neq 0$ and data; dashed lines, $\tau_{\mathrm{C} \text { II }} \neq 0$ and Si II with $v_{\max }=30 \times 10^{3} \mathrm{~km} \mathrm{~s}^{-1}$. The continuum level has been shifted to match the data. Ions responsible for features in the synthetic spectrum are labeled. The position of the minimum changes by about $50 \AA$ when the $\mathrm{C}$ II component is included. The small notch around $6200 \AA$ is an artifact of the spectrum extraction and does not have to be considered a supernova feature. weaker Si II $\lambda 6355$ absorption than SN 1999aa. This suggests that the amount of Si II present in SN 1999aa and/or the atmospheric temperature could be between that of normals and SN 1991T-like SNe Ia. The Ca II IR triplet around $8000 \AA$ has two weak features not present in SN 1981B but visible in SN 1994D. All the other major features are the same as those seen in normal SNe Ia.

\subsubsection{Synthetic Spectrum}

The synthetic spectrum for day -1 is shown in Figure 8. As Q5 in the previous epoch, $\mathrm{Si}$ II and $\mathrm{Si}$ III have been introduced with a low maximum velocity in order to match the observed wavelengths of the minima; see Table 3 for details. The S II, $\mathrm{Mg}$ II, $\mathrm{Co}$ II, and $\mathrm{Fe}$ II lines are well reproduced in detached layers.

The $\mathrm{Ca} \mathrm{H}$ and $\mathrm{K}$ feature is formed by a blend of the two dominant components of $\mathrm{Ca}$ II and weaker contributions from $\mathrm{Si}$ II, Co II, and $\mathrm{Ni}$ II. The double component of $\mathrm{Ca}$ II is required at this epoch both in the $\mathrm{H}$ and $\mathrm{K}$ and IR triplet regions (Fig. 9, left and right, respectively). The split seen at the location of $\mathrm{Ca} \mathrm{H}$ and $\mathrm{K}$ has been reproduced with Si II 23858 or Si III 23801 in non-LTE (NLTE) simulations (Nugent et al. 1997; Lentz et al. 2000) but with SYNOW, the only way to account for the blue minimum of $\mathrm{Ca} \mathrm{H}$ and $\mathrm{K}$ is to add a highvelocity component (Ca II HV; Hatano et al. 1999a). Ca II HV

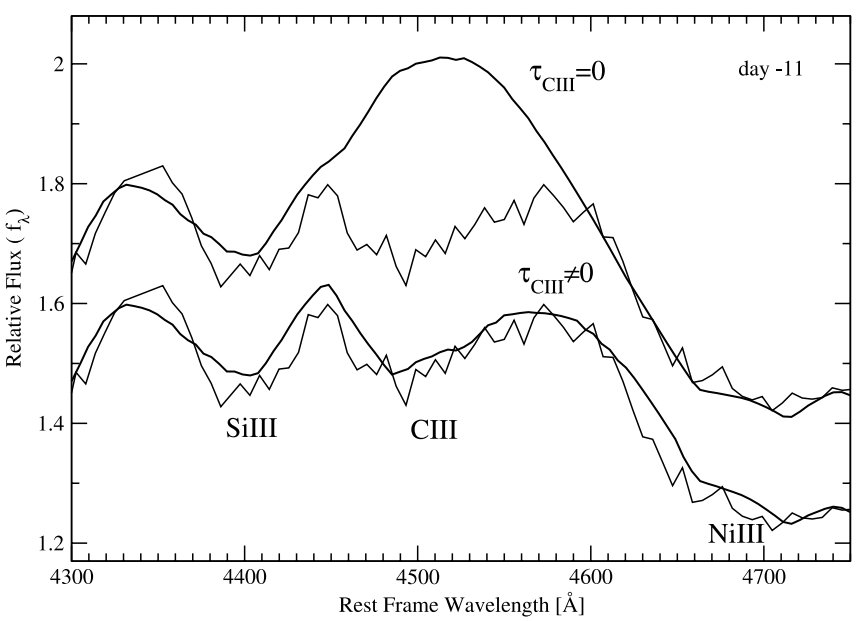

FIG. 6.-Synthetic spectra compared with SN 1999aa spectrum for day -11 around $4500 \AA$. Lines from top to bottom: $\tau_{\mathrm{C} \text { III }}=0$ and data, $\tau_{\mathrm{C} \text { III }} \neq 0$ and data. 


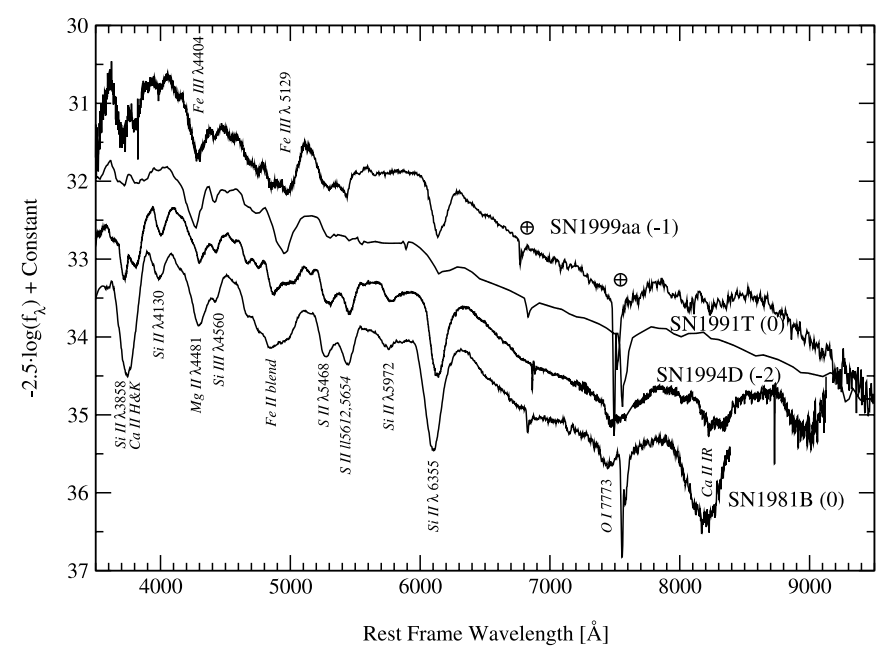

FIG. 7.-Day -1 spectrum of SN 1999aa together with those of SN 1991T, SN 1981B, and SN 1994D from Jeffery et al. (1992); Branch et al. (1983), and Patat et al. (1996). Each spectrum is labeled with the phase (days since $B$ maximum). The circled plus sign marks atmospheric absorptions.

with $v_{\text {min }}=21000 \mathrm{~km} \mathrm{~s}^{-1}$ also matches the two small absorptions in the blue part of the $\mathrm{Ca}$ II IR triplet. In the right panel of Figure 9 the blackbody continuum has been shifted to the level of the observed spectrum around $8000 \AA$ to show the match in velocity. The correct relative intensity of the two HV $\mathrm{Ca}$ II IR triplet features and the correct line profile are better reproduced by modeling the three-dimensional geometry of the HV clump (Kasen et al. 2003; Thomas et al. 2003). In this work we are only interested in identifying the velocity range at which this component is present.

The absorption features we tentatively identified as $\mathrm{C}$ II and $\mathrm{C}$ III in the early spectrum are no longer detectable. The broader and stronger Si II $\lambda 6355$ and Si III $\lambda 5051$ now dominate the wavelength ranges at which these lines were present.

As seen in Figure 8, the synthetic spectrum fails to describe the data in several wavelength regions: around 4600, 5500, and beyond $6200 \AA$. In similar analyses with SYNOW, attempts were made to improve the match around $4600 \AA$ by adding a HV component of Fe II. For SN 1999aa, this approach introduced mismatches in other wavelength regions. The disagreement, as well as that around $5500 \AA$, could also be (partly) explained by the inaccuracy of the blackbody continuum, which is clearly showing a mismatch for the flux redward of $6200 \AA$.

As for the spectrum at day -11 , the blackbody continuum fails to produce the correct flux redward of $6200 \AA$, but this does not affect the reliability of the line profile reproduction. Despite attempting several different parameter combinations, the synthetic spectrum shows significant disagreement with the data at $4600 \AA$ on the red side of $\mathrm{S}$ II.

\subsection{Day 5 After Maximum}

The spectrum of SN 1999aa around 1 week after maximum is shown in Figure 10 and compared with those of SN 1991T, SN 1994D, and SN 1990N. The spectrum of SN 1999aa is quite normal, now also showing the blue component of the $\mathrm{Si}$ II duo in the 5700-6200 $\AA$ region. SN $1991 \mathrm{~T}$ still exhibits weaker Si II and S II lines at this epoch. In normal SNe, absorption features due to elements such as $\mathrm{Co}$ II, Fe II, Ca II IR triplet, and $\mathrm{O}_{\mathrm{I}} \lambda 7773$ get stronger at this time. From Figure 10 and from the spectra of other SN 1991T-like supernovae shown in Li et al. (1999, 2001a), it is clear that the general trend for these objects at this epoch is to still have very weak IMEs components and a persistent dominant presence of doubly ionized Fe. Furthermore, SN 1997br and SN 2000cx (Li et al. 1999, 2001a) already have a large contribution from low-ionization iron-peak elements (more prominent than in Branch-normal SNe), with SN 1997br more advanced in this transition. This suggests a very interesting sequence among these objects in which the duration in the phase of the IMEs is shortest for SN 1997br and longest (and thus more similar to normal SNe) for SN 1999aa, with SN 1991T somewhere in the middle. For Branch-normal supernovae, this phase extends from well before maximum to a couple of weeks after. In the case of these peculiar objects it seems instead to vary in duration and phase during the time evolution. This is probably due to the ratio of Fe-peak to IME abundances, their radial distribution and temperature profile. Further studies are needed to quantify these differences.

\subsubsection{Synthetic Spectrum}

In the synthetic spectrum for day 5 after maximum, shown in Figure 11, doubly ionized $\mathrm{Si}$ and $\mathrm{Fe}$ have lower optical depth than in the previous epoch analyzed; see Table 4. Fe III and Si III are now blended, forming a deep trough at $4400 \AA$ together with $\mathrm{Co}$ II and $\mathrm{Mg}$ II. We also note a mismatch between the data and the synthetic spectrum around $4600 \AA$.

In order to reproduce the three distinct minima below $5000 \AA$, the $\mathrm{Fe}$ II layer has to be detached, with a similar velocity range as in day -1 , otherwise the lines tend to blend together forming a single broad feature.

At this epoch, Si II and Si III also become detached, as did $\mathrm{S}$ II and $\mathrm{Mg}$ II in the previous epoch. Now they all appear confined above $10 \times 10^{3} \mathrm{~km} \mathrm{~s}^{-1}$. This is a probable sign that complete silicon burning has stopped at this velocity.

The feature at $5700 \AA$ is a blend of $\mathrm{Na}$ I with a weak Si II component. The Si II component is weak because of the low excitation temperature used; a higher temperature would create a redder feature than the one seen in our data.

Unfortunately, at this epoch our spectrum does not cover the region below $3900 \AA$, and the Ca IR triplet is too weak

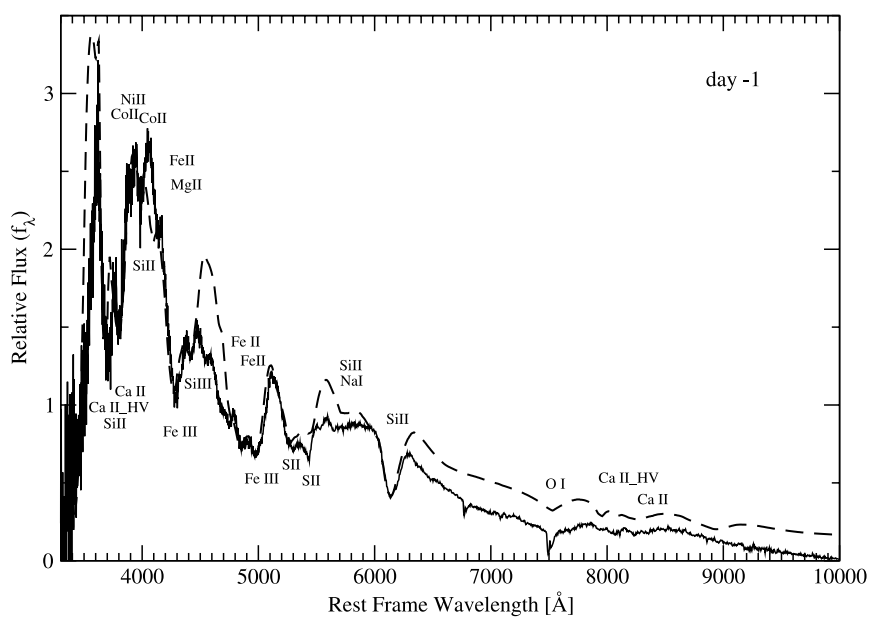

FIG. 8.-Synthetic spectrum compared with SN 1999aa spectrum for day -1 . SYNOW parameters used are presented in Table 3 . The regions around 3800 and $8000 \AA$ are enlarged in Fig. 9 to highlight the contribution of Ca II HV. Above $6200 \AA$ the blackbody assumption fails in reproducing the correct flux values, but it does not affect the identification of the lines. Ions responsible for features in the synthetic spectrum are labeled. 
TABLE 3

SYNOW PARAMETERS FOR DAY -1

\begin{tabular}{|c|c|c|c|c|c|}
\hline Ion & $\tau$ & $\begin{array}{c}v_{\min } \\
\left(10^{3} \mathrm{~km} \mathrm{~s}^{-1}\right)\end{array}$ & $\begin{array}{c}v_{\max } \\
\left(10^{3} \mathrm{~km} \mathrm{~s}^{-1}\right)\end{array}$ & $\begin{array}{c}T_{\mathrm{exc}} \\
\left(10^{3} \mathrm{~K}\right)\end{array}$ & $\begin{array}{c}v_{e} \\
\left(10^{3} \mathrm{~km} \mathrm{~s}^{-1}\right)\end{array}$ \\
\hline $\mathrm{Ca}$ II ................ & 3 & $\ldots$ & 18 & 8 & 5 \\
\hline $\mathrm{Ca}$ II $(\mathrm{HV})^{\mathrm{a}} \ldots .$. & 2.5 & 22 & 25 & 10 & 5 \\
\hline $\mathrm{O}_{\mathrm{I}} \ldots \ldots \ldots \ldots \ldots . . . . . . . . .$. & 0.2 & $\ldots$ & 30 & 8 & 5 \\
\hline Si II .................. & 0.9 & $\ldots$ & 15 & 8 & 5 \\
\hline Si III ................ & 0.25 & $\ldots$ & 15 & 8 & 5 \\
\hline 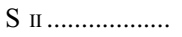 & 0.65 & 10 & 20 & 8 & 5 \\
\hline Mg II............... & 0.1 & 10 & 20 & 8 & 5 \\
\hline Fe II ................ & 1 & 10 & 15 & 8 & 5 \\
\hline Co II................. & 0.3 & 13 & 30 & 8 & 5 \\
\hline $\mathrm{Na}$ I.................. & 0.25 & $\ldots$ & 30 & 8 & 5 \\
\hline Fe $\mathrm{III} \ldots \ldots \ldots \ldots \ldots$ & 0.8 & $\ldots$ & 30 & 8 & 3 \\
\hline Ni II ................ & 0.1 & $\ldots$ & 30 & 8 & 5 \\
\hline
\end{tabular}

Note.-The fit is shown in Fig. 8; $v_{\text {phot }}=9500 \mathrm{~km} \mathrm{~s}^{-1}, T_{\mathrm{bb}}=13500 \mathrm{~K}$.

a (HV): High-velocity component.

to fully determine whether there is still need for a $\mathrm{HV}$ component.

\subsection{Day 14 after Maximum}

Our spectrum of SN 1999aa at day +14 is shown in Figure 12 together with those of SN 1991T, SN 1994D, and SN 1990N. SN 1999aa is now completely indistinguishable from a normal supernova, such as SN $1990 \mathrm{~N}$, with the possible exception of a slightly weaker $\mathrm{Si}$ II 26355 . This is to be expected, since small differences are visible even among so called normals. In comparison SN 1991T is still completing its IME phase; S II has not yet blended with $\mathrm{Na}$ I as in normal objects. The overall contribution of S II, Si II, and Ca II is consistently weaker than in typical Type Ia.

\subsubsection{Synthetic Spectrum}

In the synthetic spectrum at day +14 , shown in Figure 13, most of the lines can be identified as Fe II (Table 5). As in the previous synthetic spectrum, in order to reproduce the three different minima in the Fe II blend region (4800 ̊) we
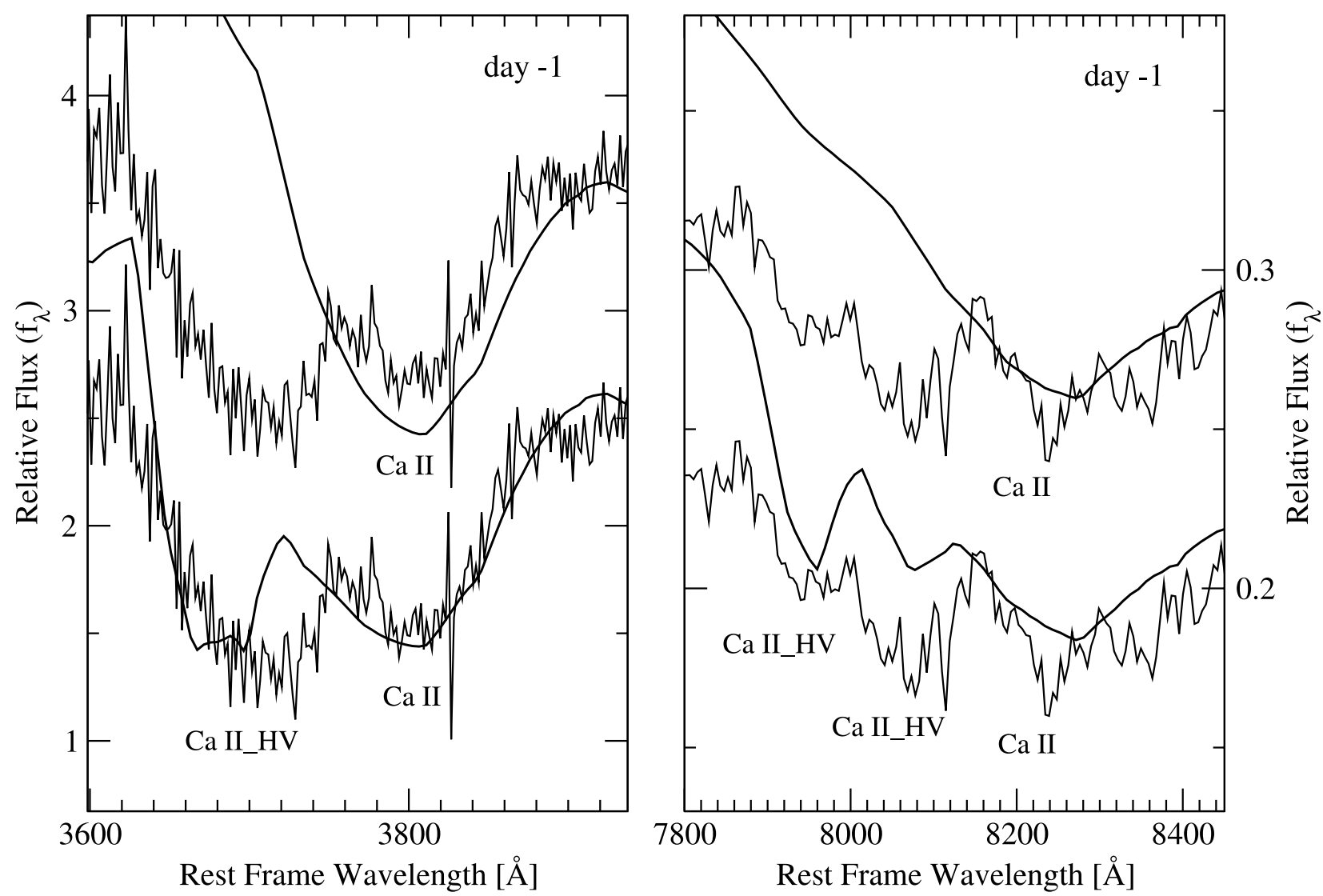

FIG. 9.-Synthetic spectrum compared with SN 1999aa spectrum for day -1 around 3800 (left) and $8000 \AA$ ( right). Top models: Solid lines, $\tau_{\mathrm{Ca}}$ I.HV $=0$ and data; Bottom models: Solid lines, $\tau_{\mathrm{Ca} \text { II HV }} \neq 0$ and data; the continuum level in the right panel has been shifted to match the data. Ions responsible for features in the synthetic spectrum are labeled. 


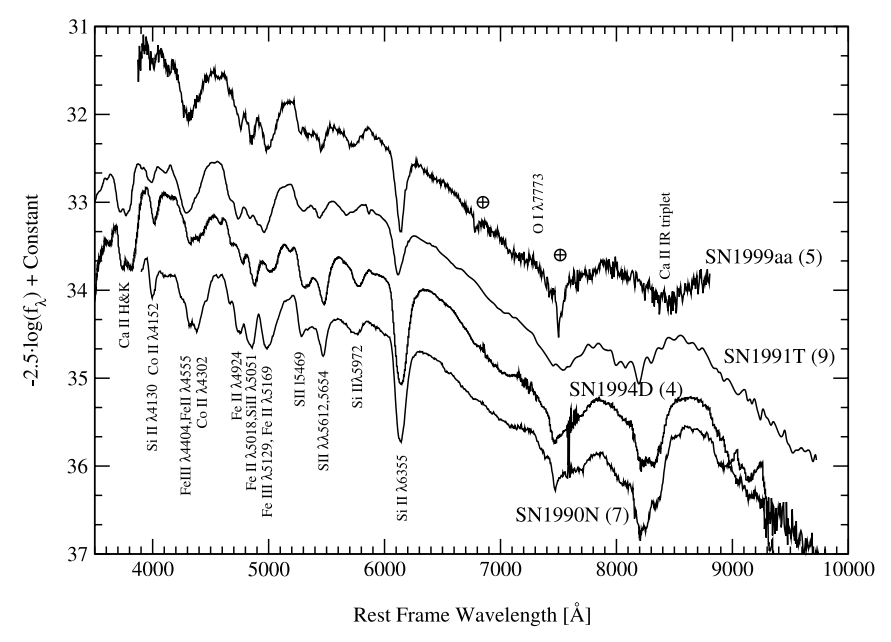

FIG. 10.-Day +5 spectrum of SN 1999aa together with those of SN 1991T, SN 1990N and SN 1994D from Filippenko et al. (1992); Leibundgut et al. (1991), and Patat et al. (1996). Each spectrum is labeled with the phase (days since B maximum). The circled plus sign marks atmospheric absorptions.

use a minimum velocity higher than that of the photosphere (Table 5).

The deep line at $5700 \AA$ is dominated by Na I, and its shape can be reproduced if $\mathrm{Na}$ I is introduced with higher $v_{e}$ than that used for the other ions.

During the entire spectral evolution, the flux level and shape in the region between 6500 and $8000 \AA$ has become increasingly more difficult to match. At this epoch, the discrepancy between data and synthetic spectrum is fairly evident. This suggests an increasing inaccuracy of the assumption of an underlying blackbody continuum.

\subsection{Early Nebular Phase Spectra}

The +25 day spectrum of SN 1999aa is shown in Figure 14. For comparison SN 1991T, SN 1994D, and SN 1981B are also shown. The main differences in the observed spectra lie in the region around $6000 \AA$, and are probably due to different $\mathrm{Si}$ abundances among the different SNe Ia. SN 1991T shows the Fe II $\lambda \lambda 6238,6246,6456$, and 6516 lines and just a very weak $\mathrm{Si}$ II line in the central part of that wavelength region. In SN 1999aa, Si II is more evident, and it becomes even stronger in SN 1994D and SN 1981B. The trough near $5700 \AA$ due to Si II $\lambda 5972$ and $\mathrm{Na}$ I D is strong in all the SNe. At the red end of the spectra, the four SNe show the typical Ca II IR triplet as a very deep and broad absorption. SN 1999aa shows a second minimum in the red part of this feature not firmly identified, but possibly consistent with an absorption feature due to Co II.

The spectrum of SN 1999aa at day +40 is shown in Figure 15 and compared with those of SN 1991T, SN 1994D, and SN 1981B. At this stage the spectra are all dominated by Fe II and Co II lines formed mainly in the deep layers of the atmosphere. Only small differences are now visible in the depths of some lines.

\subsubsection{Synthetic Spectrum}

At day +40 the supernova has started entering the so-called nebular phase and SYNOW's assumption of a sharp photosphere becomes less physically realistic. Even though SYNOW was designed to reproduce only spectra in the photospheric phase, several authors have drawn conclusions based on its matches to nebular spectra (see, e.g., Fisher et al. 1999). In the interest of comparison with their work, we also present the synthetic spectrum for this epoch.

With the photosphere now into the iron-peak core, at this epoch the supernova spectrum is mainly formed by Fe II and Co II; see Figure 16. The only signs of IMEs are $\mathrm{Ca}$ II $\mathrm{H}$ and $\mathrm{K}$ and the Ca IR triplet. As proposed by Fisher et al. (1999) for SN 1991T, the strong and wide feature near $7000 \AA$ can be reproduced by forbidden lines of $\left[\mathrm{O}_{\mathrm{II}}\right](\lambda \lambda 7320,7330)$ with a high $v_{e}$. Note that in SYNOW this line is also treated with a resonance-scattering source function. For the details of the composition, see Table 6. Special care was taken in the way the components of Fe II and Co II have been introduced. At this epoch, the spectrum is formed in the Fe-peak core but still a part of the outer atmosphere makes a contribution. The optical depth of these elements in the two regions can be different.

Mazzali et al. (1998) demonstrated that the expansion velocity of the Fe-peak core and the luminosity of SNe Ia are correlated. Slow decliner supernovae, such as SN 1991T, are expected to have a higher Fe-peak core limit velocity and thus a larger region dominated by nuclear statistical equilibrium. In this respect, it is interesting to attempt the identification of the iron-peak core limit by means of SYNOW synthetic spectra. Fisher et al. (1999) and Hatano et al. (2002) introduced an optical depth discontinuity for Co II and Fe II at 10,000 and $12,500 \mathrm{~km} \mathrm{~s}^{-1}$ for their synthetic spectra of SN $1991 \mathrm{~T}$ and SN 1997br, respectively. The velocity at which this change occurs can be thought of as the iron-peak core limit. We tried to reproduce this for SN 1999aa. The best match was achieved with a discontinuity at $10,000 \mathrm{~km} \mathrm{~s}^{-1}$.

\section{EXPANSION VELOCITIES}

The expansion velocities as computed from fits to the minima of the spectral lines can provide help in investigating the physics of the supernova explosion. Weighted fits of the observed minima were performed using a nonlinear Marquardt-Levenberg minimization procedure (Marquardt 1963) applied to a Gaussian profile model. The minimum of the line was considered to be the center of the Gaussian and the fit uncertainty its statistical error. This statistical error is usually on the order of a few kilometers per second, so it is not shown on the graphs. In general, the fit was performed on the entire

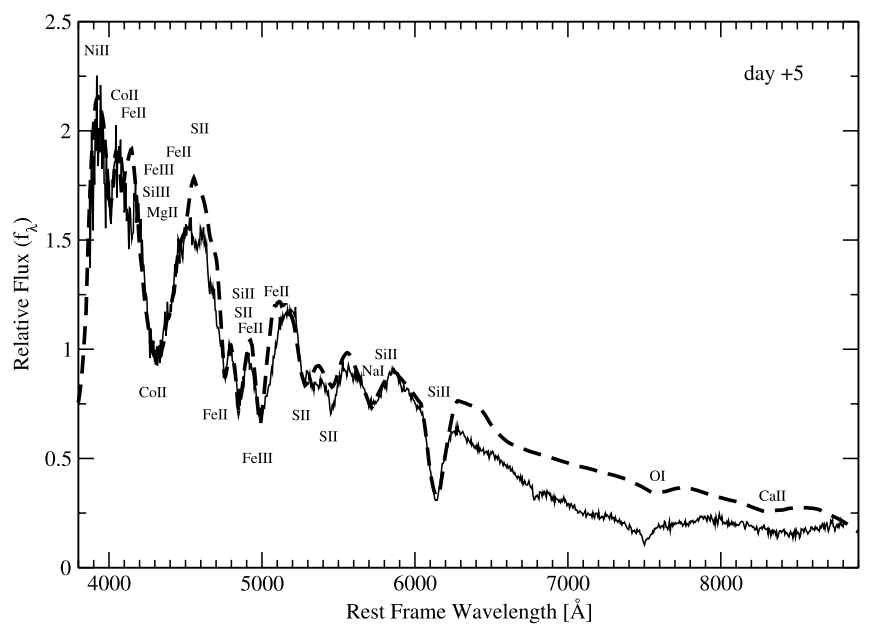

FIG. 11.- Synthetic spectrum compared with SN 1999aa spectrum for day +5 . SYNOW parameters used are presented in Table 4 . Above $6200 \AA$ the blackbody assumption fails in reproducing the correct flux values, but it does not affect the identification of the lines. Ions responsible for features in the synthetic spectrum are labeled. 
TABLE 4

SYNOW PARAMETERS FOR DAY +5

\begin{tabular}{|c|c|c|c|c|c|}
\hline Ion & $\tau$ & $\begin{array}{c}v_{\min } \\
\left(10^{3} \mathrm{~km} \mathrm{~s}^{-1}\right)\end{array}$ & $\begin{array}{c}v_{\max } \\
\left(10^{3} \mathrm{~km} \mathrm{~s}^{-1}\right)\end{array}$ & $\begin{array}{c}T_{\mathrm{exc}} \\
\left(10^{3} \mathrm{~K}\right)\end{array}$ & $\begin{array}{c}v_{e} \\
\left(10^{3} \mathrm{~km} \mathrm{~s}^{-1}\right)\end{array}$ \\
\hline $\mathrm{Ca}$ II ........... & 20 & $\ldots$ & 30 & 5 & 3 \\
\hline $\mathrm{O}_{\mathrm{I}} \ldots \ldots \ldots \ldots$ & 0.1 & $\ldots$ & 30 & 5 & 3 \\
\hline Si II ............ & 1.5 & 10 & 15 & 5 & 3 \\
\hline Si III ........... & 0.05 & 10 & 15 & 5 & 3 \\
\hline $\mathrm{S}$ II ............. & 0.45 & 10 & 20 & 5 & 3 \\
\hline Mg II......... & 0.6 & 10 & 20 & 5 & 3 \\
\hline Fe II ........... & 1.3 & 11 & 14 & 5 & 3 \\
\hline Co II........... & 0.8 & 11.5 & 30 & 5 & 3 \\
\hline $\mathrm{Na}$ I............ & 0.4 & $\ldots$ & 30 & 5 & 5 \\
\hline Fe III .......... & 0.09 & $\ldots$ & 30 & 5 & 3 \\
\hline Ni II ........... & 0.2 & $\ldots$ & 30 & 5 & 3 \\
\hline
\end{tabular}

Note.-The fit is shown in Fig. $11 ; v_{\text {phot }}=9000 \mathrm{~km} \mathrm{~s}^{-1}, T_{\mathrm{bb}}=12,000 \mathrm{~K}$.

absorption of the P Cygni profile when this was well reproduced by a Gaussian model, e.g., when there was a low level of contamination by other lines. In the contaminated cases the fit was done on a sample closer to the bottom of the line. In Figures 17-20 we show the time evolution of the velocities for $\mathrm{Ca}$ II $\mathrm{H}$ and $\mathrm{K}, \mathrm{Si}$ II $\lambda 6355$, and $\mathrm{Fe}$ III $\lambda \lambda 4404,5129$ lines for SN 1999aa along with several other supernovae from the literature.

Ca II H and K velocities for SN 1999aa, shown in Figure 17, are in the range of those of normal SNe. This feature shows an evident split in the minimum at days -3 and -1 . In these cases the measurements have been performed selecting a wavelength range that covers the whole line profile from approximately 3600 to $3850 \AA$. This was chosen in order to obtain a value of the velocity that could be compared with the other supernovae. Measuring the two minima and deriving from those the velocities of the two components of $\mathrm{Ca}$ II used in the synthetic spectrum would not be appropriate, since the position of the troughs are the result of the blending of $\mathrm{Ca}$ II, Si II, Co II, and Ni II.

Si II velocities are shown in Figure 18. If the absorption near $6150 \AA ̊$ of SN 1999aa was due only to Si II 26355 , the velocities would have been monotonically decreasing with time, as

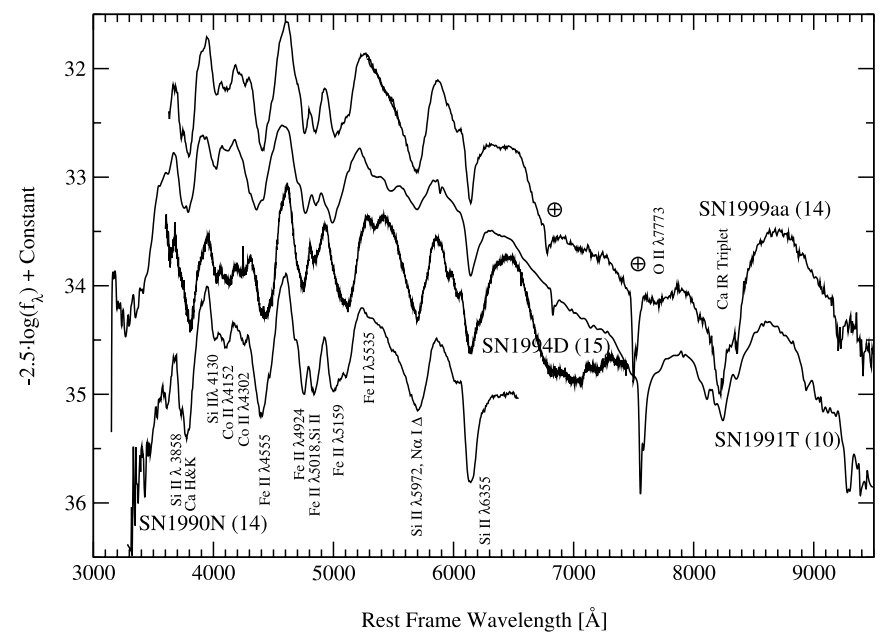

FIG. 12.-Day +14 spectrum of SN 1999aa together with those of SN 1991T, SN 1990N, and SN 1994D from Phillips et al. (1992), Leibundgut et al. (1991), and Mazzali et al. (1993). Each spectrum is labeled with the phase (days since B maximum). The circled plus sign marks atmospheric absorptions. is seen for the normal SN 1994D, SN 1992A, and the subluminous SN 1999by and SN 1991bg. For SN 1999aa the first point, 11 days before maximum light, has the lowest velocity. This is consistent with another ion (probably $\mathrm{C}$ II or $\mathrm{H}$ according to the SYNOW fit, see $\S \S 4.1$ and 6.3) also being responsible for the absorption feature at this epoch. The wavelength of the line minimum remains practically constant during the first 20 days after maximum. This is usually interpreted as if the element layer in the supernova atmosphere is confined to a region above the photosphere, in this case around $10,100 \mathrm{~km} \mathrm{~s}^{-1}$ as confirmed in the SYNOW synthetic spectra. The same trend is shown by SN 2000cx and SN 1991T, with velocities around 12,000 and $9400 \mathrm{~km} \mathrm{~s}^{-1}$, respectively, as indicated by the dashed horizontal lines in Figure 18.

The velocities measured for the two Fe III features are shown in Figures 19 and 20. These lines are characteristic of the premaximum spectra of the SN 1991T-like SNe Ia and disappear within the first week after maximum light. In both cases SN 1999aa shows lower velocities compared to SN 1991T, SN 1997br, and SN 2000cx, although all have a similar slope. Fe III $\lambda 4404$ velocities (Fig. 19) are smaller by $2500 \mathrm{~km} \mathrm{~s}^{-1}$ with respect to SN 1991T, and $3500 \mathrm{~km} \mathrm{~s}^{-1}$ with respect to SN 2000cx. Fe III $\lambda 5129$ velocities (Fig. 20)

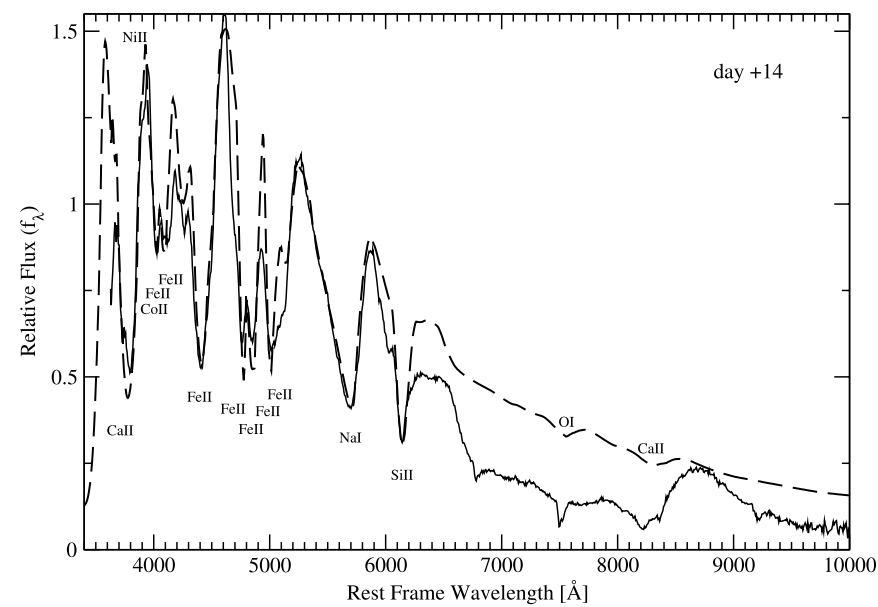

FIG. 13.- Synthetic spectrum compared with SN 1999aa spectrum for day +14 . SYNOW parameters used are presented in Table 5. Above $6200 \AA$ the blackbody assumption fails in reproducing the correct flux values, but it does not affect the identification of the lines. Ions responsible for features in the synthetic spectrum are labeled. 
TABLE 5

SYNOW PARAMETERS FOR DAY +14

\begin{tabular}{|c|c|c|c|c|c|}
\hline Ion & $\tau$ & $\begin{array}{c}v_{\min } \\
\left(10^{3} \mathrm{~km} \mathrm{~s}^{-1}\right)\end{array}$ & $\begin{array}{c}v_{\max } \\
\left(10^{3} \mathrm{~km} \mathrm{~s}^{-1}\right)\end{array}$ & $\begin{array}{c}T_{\text {exc }} \\
\left(10^{3} \mathrm{~K}\right)\end{array}$ & $\begin{array}{c}v_{e} \\
\left(10^{3} \mathrm{~km} \mathrm{~s}^{-1}\right)\end{array}$ \\
\hline $\mathrm{Ca}$ II....... & 20 & $\ldots$ & 30 & 5 & 3 \\
\hline $\mathrm{O}_{\mathrm{I}} \ldots . . . .$. & 0.1 & $\ldots$ & 30 & 5 & 3 \\
\hline Si II ........ & 1.5 & 10 & 14 & 5 & 3 \\
\hline Fe II ....... & 4 & 9.5 & 13.5 & 5 & 3 \\
\hline Co II....... & 1.7 & 10 & 30 & 5 & 3 \\
\hline $\mathrm{Na}$ I........ & 1.2 & $\ldots$ & 30 & 5 & 5.5 \\
\hline Ni II ....... & 5 & $\ldots$ & 10 & 5 & 3 \\
\hline
\end{tabular}

Note. - The fit is shown in Fig. $13 ; v_{\text {phot }}=8500 \mathrm{~km} \mathrm{~s}^{-1}, T_{\mathrm{bb}}=10500 \mathrm{~K}$.

are still smaller, but much closer to the velocities of the other peculiar supernovae. This could be due to a higher contamination from $\mathrm{Si}$ II $\lambda 5051$ or $\mathrm{Fe}$ II $\lambda 5018$.

Generally, the velocity ranges of the lines of SN 1999aa are consistent with those of normal supernovae. The major peculiarity is the restricted atmosphere region where $\mathrm{Si}$ II is present, which makes this ion appear clearly only 1 week before maximum light.

\section{DISCUSSION}

The analysis carried out in $\S 4$ indicates that SN 1999aa was a peculiar object prior to maximum light, developing toward normal-looking spectra around maximum. The SYNOW synthetic spectra have shown the velocity ranges in which each adopted ion appears, and offer a model of the structure of the expanding atmosphere. In this section we summarize our findings for different ions and their velocity distributions and discuss the consequences for constraining the progenitor system and the explosion models.

\subsection{Intermediate Mass Elements}

The velocity range in which IMEs are found constrains the atmospheric region in which incomplete $\mathrm{Si}$ burning takes place, and thus the hydrodynamics of the supernova explosion. We identify the contributions of ions such as $\mathrm{Si}$ II, S II, $\mathrm{Ca}$ II, $\mathrm{Na}$ I, and $\mathrm{Mg}$ II, which are common to both normal and

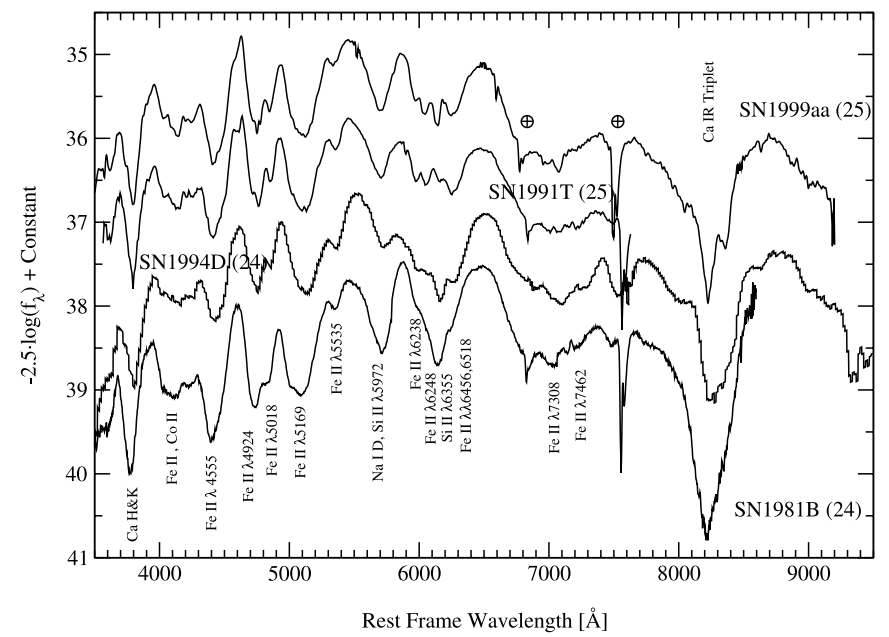

FIG. 14. - Day +25 spectrum of SN 1999aa together with those of SN 1991T, SN 1981B, and SN 1994D from Phillips et al. (1992), Branch et al. (1983), and Patat et al. (1996). Each spectrum is labeled with the phase (days since $B$ maximum). The circled plus sign atmospheric absorptions.

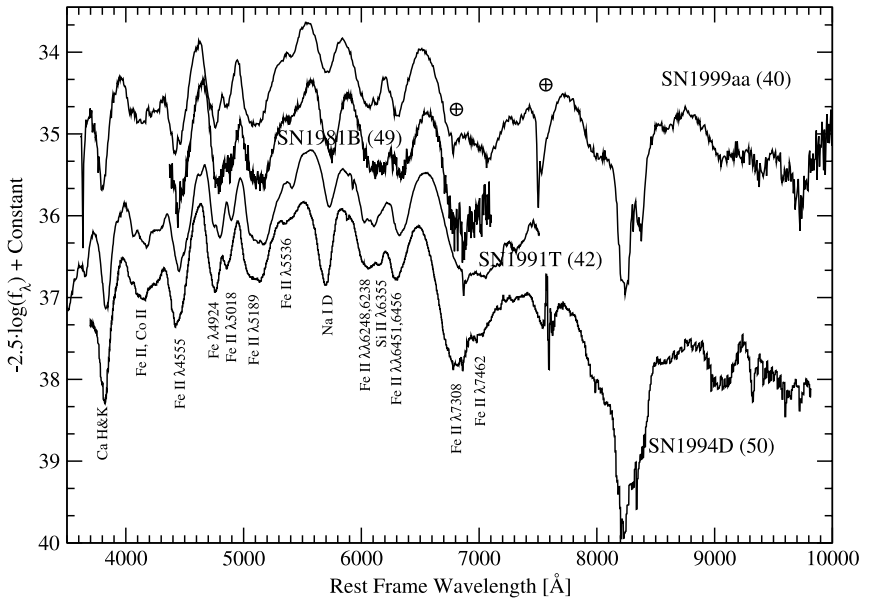

Fig. 15.-Day +40 day spectrum of SN 1999aa together with those of SN 1991T, SN 1981B, and SN 1994D from Gomez et al. (1996), Branch et al. (1983), and Patat et al. (1996). Each spectrum is labeled with the phase (days since B maximum). The circled plus sign marks atmospheric absorptions.

SN 1991T-like supernovae. However, the optical depths and velocity ranges found for these elements for SN 1999aa differ from those typically used for Branch-normal SNe. The presence of unburned $\mathrm{C}$ and IMEs (HV Ca II and possible signs of C II) above $14,000 \mathrm{~km} \mathrm{~s}^{-1}$, as found in our synthetic spectra of SN 1999aa, suggests that this supernova was not the result of a sub-Chandrasekhar mass explosion, as the composition resulting from the modeling of such explosions does not include any IMEs or $\mathrm{C}$ in the external layer of the envelope (see, e.g., Livne \& Arnett 1995; Woosley \& Weaver 1994).

The time evolution of the Doppler blueshift of Si II, Figure 18, around and after $B$-band maximum shows that this ion is confined within a narrow velocity range $\left(v_{\min } \sim\right.$ $\left.10,100 \mathrm{~km} \mathrm{~s}^{-1}\right)$, not only for SN 1999aa but also in SN 2000cx $\left(v_{\min } \sim 12,000 \mathrm{~km} \mathrm{~s}^{-1}\right)$ and SN 1991T $\left(v_{\min } \sim 9400 \mathrm{~km} \mathrm{~s}^{-1}\right)$. Our SYNOW models of SN 1999aa provide independent evidence of a confined Si II layer. Velocities between 10,000 and $15,000 \mathrm{~km} \mathrm{~s}^{-1}$ were needed to match the observations when the photospheric velocity dropped below $9500 \mathrm{~km} \mathrm{~s}^{-1}$ (see Tables 2-6). The same holds for SN 1997br (Hatano et al.

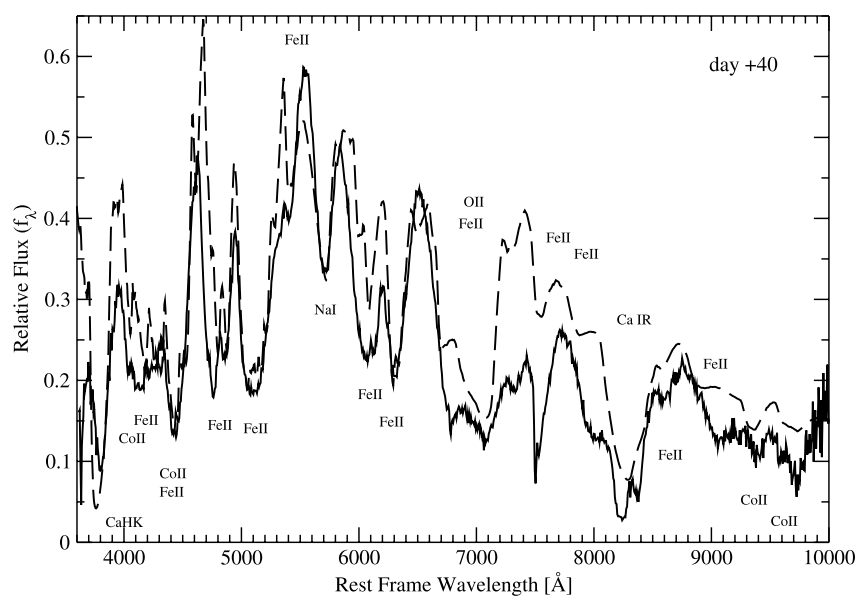

FIG. 16.-Synthetic spectrum compared with SN 1999aa spectrum for day +40 . SYNOW parameters used are presented in Table 6 . A discontinuity at $10,000 \mathrm{~km} \mathrm{~s}^{-1}$ has been introduced in the Co and Fe optical depths, indicating the possible iron-peak core limit. Ions responsible for features in the synthetic spectrum are labeled. 
TABLE 6

SYNOW PARAMETERS FOR DAY +40

\begin{tabular}{|c|c|c|c|c|c|}
\hline Ion & $\tau$ & $\begin{array}{c}v_{\min } \\
\left(10^{3} \mathrm{~km} \mathrm{~s}^{-1}\right)\end{array}$ & $\begin{array}{c}v_{\max } \\
\left(10^{3} \mathrm{~km} \mathrm{~s}^{-1}\right)\end{array}$ & $\begin{array}{c}T_{\mathrm{exc}} \\
\left(10^{3} \mathrm{~K}\right)\end{array}$ & $\begin{array}{c}v_{e} \\
\left(10^{3} \mathrm{~km} \mathrm{~s}^{-1}\right)\end{array}$ \\
\hline Ca II ........... & 300 & $\ldots$ & 18 & 8 & 3 \\
\hline $\mathrm{O}$ II ............... & 1 & $\ldots$ & 30 & 10 & 10 \\
\hline Fe II ............ & 160 & $\ldots$ & 10 & 5 & 3 \\
\hline Fe II ........... & 0.2 & 10 & 13.5 & 5 & 3 \\
\hline Co II........... & 60 & $\ldots$ & 10 & 5 & 3 \\
\hline Co II........... & 0.2 & 10 & 30 & 5 & 3 \\
\hline Na I............ & 0.9 & $\ldots$ & 30 & 5 & 5 \\
\hline
\end{tabular}

Note.-The fit is shown in Fig. $4 ; v_{\text {phot }}=8000 \mathrm{~km} \mathrm{~s}^{-1}, T_{\mathrm{bb}}=8000 \mathrm{~K}$.

2002) and for SN 1991T (Fisher et al. 1999). For a normal SNe Ia, such as SN 1994D, the Si-rich layer extends from the photosphere out to $v>25,000 \mathrm{~km} \mathrm{~s}^{-1}$ (Hatano et al. 1999a). For SN 1991T-like or SN 1999aa-like objects, the weakness of the IMEs' absorption and the earlier than normal domination of the Fe-group elements suggest that the region of incomplete Si-burning could be confined to a small velocity window. Thus, a higher deflagration to detonation transition density in DD models could explain some of the spectral peculiarities.

In DD hydrodynamic models this would imply an overluminous object. We do not have a sufficiently accurate absolute magnitude measurement of SN 1999aa, but if the light curve-width luminosity relation holds for this object, SN 1999aa should be more luminous than normal SNe. Based on the measurement of the distance to SN 1991T, Saha et al. (2001) and Gibson \& Stetson (2001) showed that this supernova is not necessarily overluminous. Thus, the possible correlations between the absolute magnitude of $\mathrm{SNe}$ Ia and the velocity range in which IMEs are present requires further studies.

\subsection{Nickel and Other Doubly Ionized Elements}

The ionization level of each component of the atmosphere in $\mathrm{SNe} \mathrm{Ia}$ is an indicator of the energy balance during the supernovae explosion. Both thermal and nonthermal ionization are known to be important in $\mathrm{SNe}$ Ia (see, e.g., Lucy 1991; Nugent et al. 1995; Baron et al. 1996).

We have found evidence of $\mathrm{Si}$ III, Fe III, and $\mathrm{Ni}$ III in the early spectra of SN 1999aa. As discussed in Nugent et al.

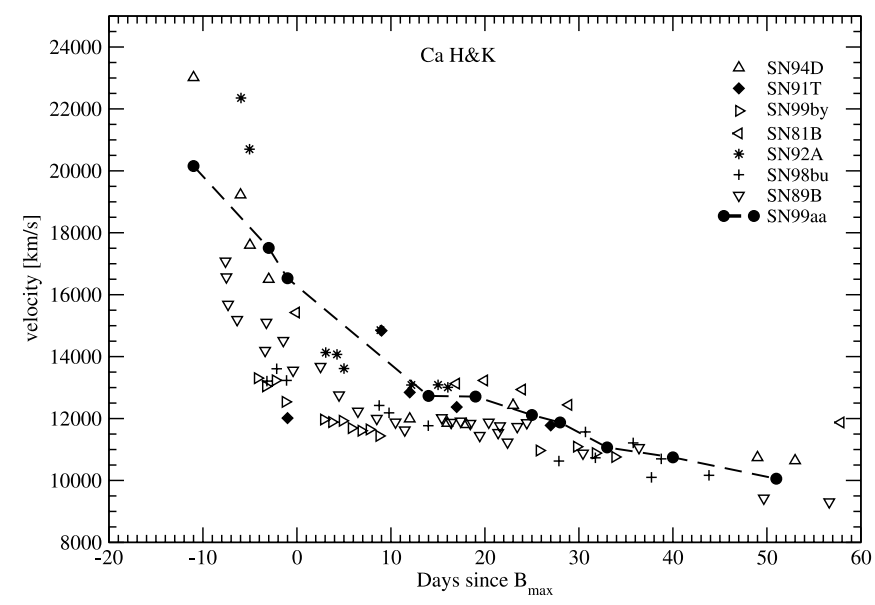

Fig. 17.-Doppler shift of the Ca H and K. The values for SN 1999aa are compared with those for other SNe Ia taken from Wang et al. (1996), Garnavich et al. (2001), Kirshner et al. (1993), Patat et al. (1996), and Jha et al. (1999) and references therein.
(1995), doubly ionized Fe and Si in the early spectra suggest higher temperatures than in Branch-normal SNe. The absorption features from these ions disappear after day +5 , as the supernova atmosphere cools.

The innermost layers of deflagration models, such as W7, are considered to have gone through complete silicon burning, leaving only a nuclear statistical equilibrium composition such as that shown in Hatano et al. (1999b). The decay of the resulting ${ }^{56} \mathrm{Ni}$ to ${ }^{56} \mathrm{Co}$ and then to stable ${ }^{56} \mathrm{Fe}$ would imply the presence of $\mathrm{Co}$ III lines not found in the present analysis. As in Hatano et al. (2002), we suggest that the Ni III lines in our premaximum spectra are produced by ${ }^{54} \mathrm{Fe}$ and ${ }^{58} \mathrm{Ni}$ synthesized during incomplete and complete silicon burning. If this is the case, it is not necessary to produce a model that extends the complete silicon burning into regions of intermediate mass elements or above, as originally proposed for SN 1991T-like objects. Among the current DD models, none is able to extend the presence of these ions sufficiently far out $\left(v_{\max }=30,000 \mathrm{~km} \mathrm{~s}^{-1}\right.$; Table 2$)$ to match our observations.

Furthermore, the measurements of the Doppler shift of Fe III, Figures 19 and 20, show a lower value than other SN 1991T-like objects. This suggests that Fe III extends further out in SN 1999aa than in spectroscopically normal SNe Ia, but has its maximal absorption at lower radii than other SN 1991Tlike objects.

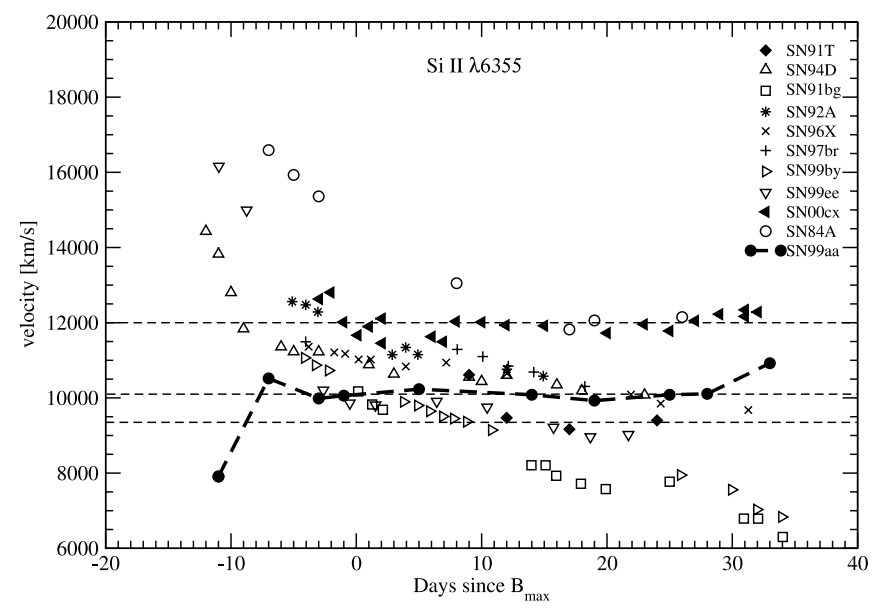

FIG. 18.-Doppler shift of the Si II $\lambda$ 6355. The values for SN 1999aa are compared with those for other SNe Ia taken from Li et al. (1999, 2001a), Garnavich et al. (2001), and Salvo et al. (2001) and references therein. The low value of SN 1999aa at day -11 is probably due to C II or H contamination. SN 2000cx, SN 1999aa and SN 1991 T maintain a constant velocity of $12,000,10,100$, and $9400 \mathrm{~km} \mathrm{~s}^{-1}$ (dashed lines), respectively, from maximum light to after day 20. 


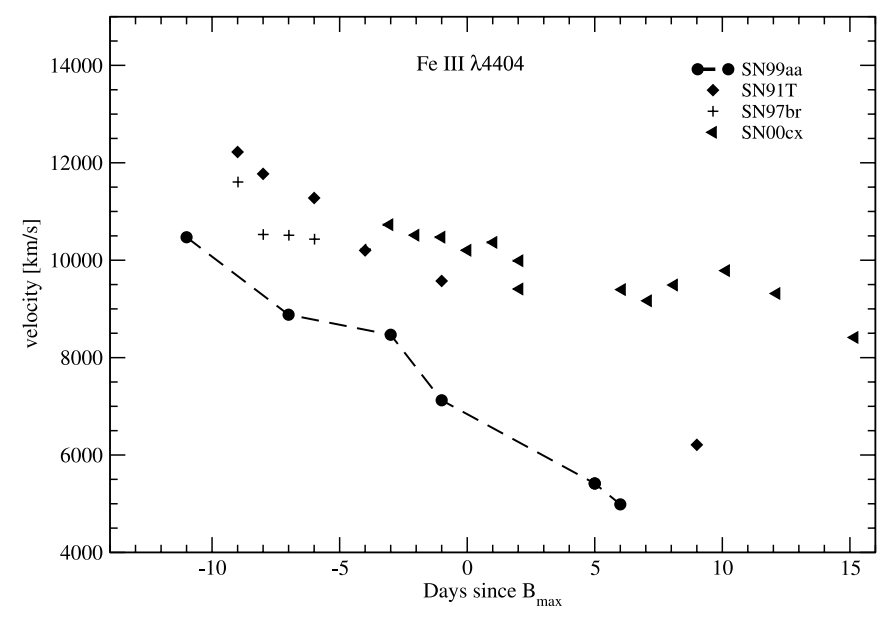

FIG. 19.-Doppler shift of the Fe III $\lambda 4404$. The values for SN 1999aa are compared with those for other SNe Ia taken from Li et al. (1999, 2001a) and references therein.

Hoeflich et al. (1998) showed that a higher initial metallicity would increase the abundance of ${ }^{54} \mathrm{Fe}$ and ${ }^{58} \mathrm{Ni}$, and this could possibly explain our observations. UV photometry and spectroscopy would yield important information to settle this issue and aid in the development of new models that could better match the observations (Lentz et al. 2000).

\subsection{II or $\mathrm{H}$ ?}

The identification of $\mathrm{H}$ or $\mathrm{C}$ would impose constraints on either the nature of the progenitor system or the hydrodynamics of the explosion, respectively. In $\S 4.1$ we suggested the presence of an HV C (C II) component for the spectrum of SN 1999aa at day -11 . In what follows, we discuss possible alternative explanations, assuming an additional ion is necessary to improve the matching.

The evidence for an external layer of carbon in SNe Ia has been discussed in the case of SN 1991T by Fisher et al. (1997, 1999), for SN 1990N by Mazzali (2001), for SN 1994D by Hatano et al. (1999a), and recently for SN 1998aq by Branch et al. (2003b), based mainly on the possible presence of C II absorption on the red side of Si II 26355 . For SN 1999aa, this ion helps in reproducing the line shape and would explain the velocity time evolution of the feature at $6150 \AA$, as shown in Figures 4 and 5 and Figure 18 ( $(5)$. However, due to the small optical depth for $\mathrm{C}_{\text {II }}(\tau=0.01$, Table 2$)$ the $\mathrm{C}_{\text {II }} 27234$ line, which could confirm the presence of $\mathrm{C}$, is too weak in the synthetic spectrum to be positively identified in the spectrum observed at day -11 .

Our identification of an external layer of C in SN 1999aa remains tentative (based only on the contribution at $6150 \AA$ ), but seems plausible given the work of other authors on early spectra of SN 1991T-like and Branch-normal SNe. The presence of $\mathrm{C}$ would point to explosion hydrodynamics consistent with pure deflagration of a Chandrasekhar mass WD (e.g., Nomoto et al. 1984).

Lentz et al. (2002) discussed the possibility that the absorption redward of Si II $\lambda 6355$ could be attributed to hydrogen mixed into the external layer from the WD companion. They modeled the presence of solar composition material mixed in the unburned $\mathrm{C}+\mathrm{O}$ layer of $\mathrm{W} 7$ to study the effects of different mixing depths. In some configurations they found that $\mathrm{H} \alpha$ could be easily mistaken for $\mathrm{C}$ II $\lambda 6580$. We have explored this possibility assuming the applicability

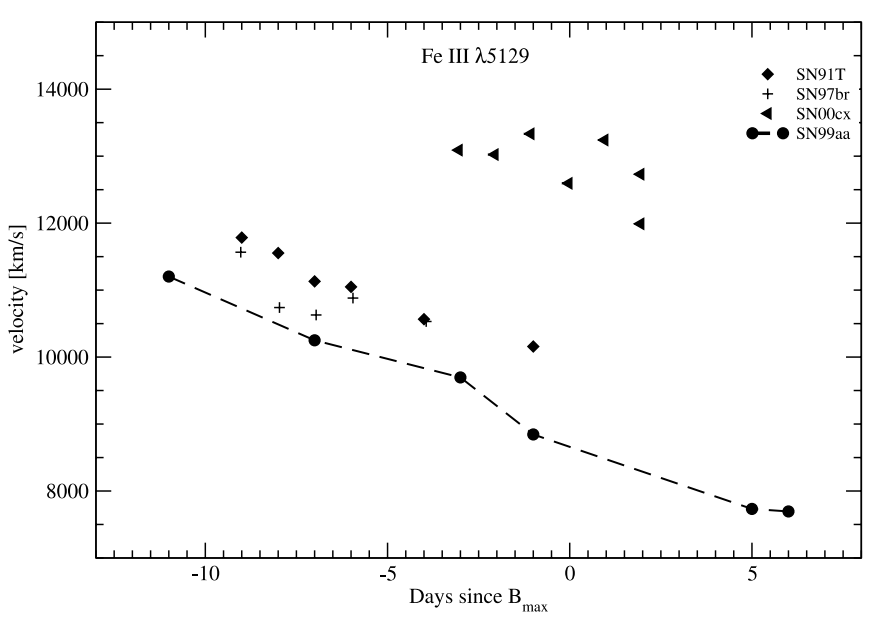

Fig. 20.-Doppler shift of the Fe III 25129 . The values for SN 1999aa are compared with those for other SNe Ia taken from Li et al. $(1999,2001 \mathrm{a})$ and references therein.

of the resonance-scattering approximation, as illustrated in Figure 21.

The continua of the three models proposed have been shifted (adding an arbitrary constant) to match the data. The top model of Figure 21 includes only Si II and, as noted in $\S 4.1$, the minimum velocity is set by the photosphere velocity, which is constrained by other ions to be $v_{\text {phot }}=11 \times 10^{3} \mathrm{~km} \mathrm{~s}^{-1}$ and cannot be changed without adversely affecting the overall match. The model in the middle reproduces better the line profile thanks to the contribution of the $\mathrm{H} \alpha$ line. The parameters used for $\mathrm{H}$ are $v_{\min }=18 \times 10^{3} \mathrm{~km} \mathrm{~s}^{-1}, v_{\max }=30 \times 10^{3} \mathrm{~km}$ $\mathrm{s}^{-1}, \tau=0.08, T_{\text {exc }}=15 \times 10^{3} \mathrm{~K}$, and $v_{e}=5 \times 10^{3} \mathrm{~km} \mathrm{~s}^{-1}$. The optical depth used does not form any visible $\mathrm{H} \beta$ line around $4500 \AA$, which is in agreement with our observations. Note, however, that for resonance scattering the net emission phenomenon is neglected, and this could change the relative strength of $\mathrm{H} \alpha$ and $\mathrm{H} \beta$ (see, e.g., Baron et al. 2000; Thomas et al. 2003). The reproduction of the line profile at $6150 \AA$ is indeed as good for $\mathrm{H} \alpha$ as for $\mathrm{C}$ II (Fig. 21, bottom). The

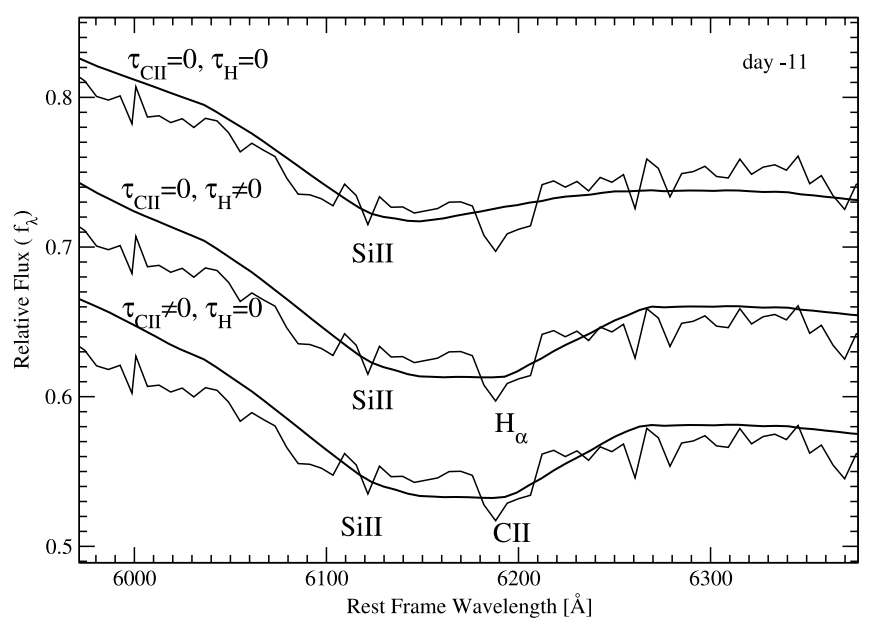

Fig. 21.-Synthetic spectra compared with SN 1999aa spectrum for day -11 around $6150 \AA \AA$. Top model: Solid lines, $\tau_{\mathrm{H}}=0, \tau_{\mathrm{C} \text { II }}=0$ and data. Middle model: Solid lines, $\tau_{\mathrm{H}} \neq 0, \tau_{\mathrm{C}} \mathrm{II}=0$ and data. Bottom model: Solid lines, $\tau_{\mathrm{C} \text { II }} \neq 0, \tau_{\mathrm{H}}=0$ and data. The continuum level has been shifted to match the data. SYNOW parameters for $\mathrm{C}$ II are presented in Table 2 and those for $\mathrm{H}$ are presented in the text. Ions responsible for features in the synthetic spectrum are labeled. 

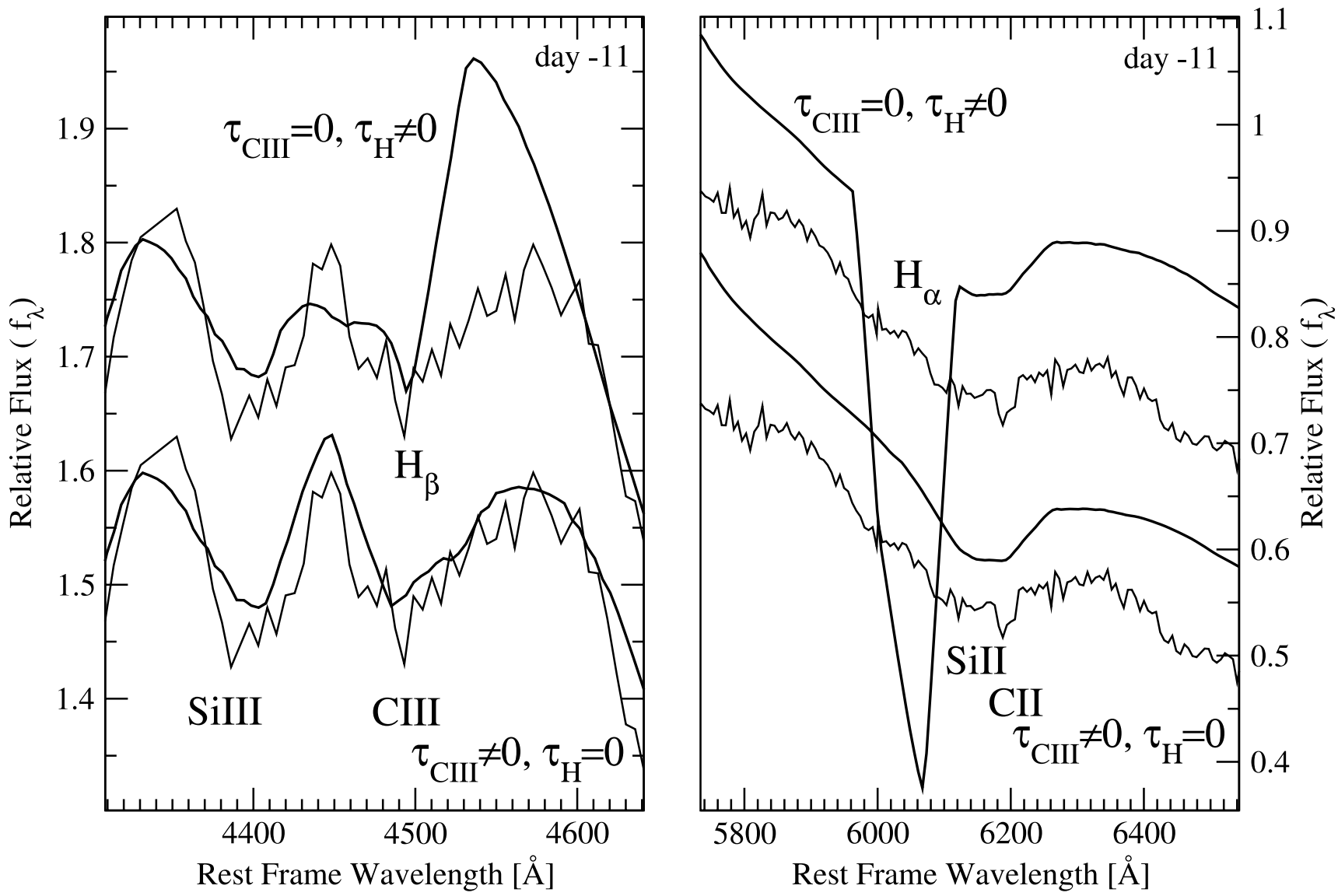

FIG. 22. - Synthetic spectra compared with SN 1999aa spectrum for day -11 around $4500($ left $)$ and $6150 \AA$ (right). Top model: Solid lines, $\tau_{\mathrm{H}} \neq 0, \tau_{\mathrm{C}}$ III $=0$ and data. Bottom model: Solid lines, $\tau_{\mathrm{H}}=0, \tau_{\mathrm{C} \text { III }} \neq 0$ (as bottom model in Fig. 6) and data. In the resonance scattering approximation the optical depth necessary to reproduce the observed absorption at $4500 \AA$ with $\mathrm{H} \beta$ produces a too strong $\mathrm{H} \alpha$ that could be weaker considering the net emission effect. SYNOW parameters for $\mathrm{C}$ III are presented in Table 2 and those for $\mathrm{H}$ are presented in the text. Ions responsible for features in the synthetic spectrum are labeled.

identification of a given ion in a supernova atmosphere based on the evidence of a single line is not definitive; therefore, we consider the contribution from either of the two ions equally plausible.

The presence of hydrogen in the external layers of the exploding WD would indicate that a single degenerate progenitor system scenario is responsible for at least some of the observed SNe Ia.

\subsection{Carbon III or Hydrogen?}

In $\S 4.1$ we included $C_{\text {III }} \lambda 4648.8$ at photospheric velocities to match the line at $4500 \AA$. The presence of unburned material deep in the IME atmospheric region is seen in three-dimensional deflagration models, in which convective flows are shown to continuously refuel the inner layers with material that has original composition (Gamezo et al. 2003). Finding unburned material deep in the atmosphere is then theoretically possible.

The first attempt to identify this line as $\mathrm{C}$ III was performed by Hatano et al. (2002), using the early spectra of SN 1997br, but they could not match the central wavelength of the line. They alternatively mentioned the possibility that $\mathrm{He}$ II could be responsible for the absorption. However, because of the high ionization energy of helium this could only be the case if there was a large degree of nonthermal ionization from the decay products of radioactive elements.

Thomas et al. (2003) tentatively suggested the possibility that the same line in SN 2000cx could be matched by an HV clump of $\mathrm{H}$. In our spectrum at day -11 prior to maximum this would not be possible because the optical depth necessary to match the line at $4500 \AA$ with $\mathrm{H} \beta$ would produce $\mathrm{H} \alpha$ in the $\mathrm{Si}$ II $\lambda 6355$ region, which is ruled out by the data as shown in Figure 22 (SYNOW parameters for H: $v_{\min }=24.5 \times$ $10^{3} \mathrm{~km} \mathrm{~s}^{-1}, v_{\max }=30 \times 10^{3} \mathrm{~km} \mathrm{~s}^{-1}, \tau=2.5, T_{\mathrm{exc}}=15 \times 10^{3} \mathrm{~K}$ and $v_{e}=3 \times 10^{3} \mathrm{~km} \mathrm{~s}^{-1}$ ). However, note that considering the net emission effect would weaken the $\mathrm{H} \alpha$ absorption feature. Furthermore, as pointed out in Thomas et al. (2003), because of the lack of a strong emission in the $6150 \AA$ region, the identification of the line at $4500 \AA$ as $\mathrm{H} \beta$ would be contingent upon a clumpy geometry and thus requires a threedimensional simulation to be fully explored.

Because of the good line profile fit, $\mathrm{C}$ III remains our best candidate for the identification of the line at $4500 \AA$ in the early spectra of SN 1999aa. Such a possibility would strongly favor current three-dimensional deflagration models of $\mathrm{C}+\mathrm{O}$ WD since they produce this ion at all velocities (Gamezo et al. 2003; Baron et al. 2003; Khokhlov 2000). Further confidence in this identification will require detailed radiative transfer modeling.

\subsection{High-Velocity $\mathrm{Ca}$ II}

The identification of HV burned material in supernova spectra can place constraints on the hydrodynamics of the explosion. The presence of a $\mathrm{HV}$ component of $\mathrm{Ca}$ II is strongly supported by the blue components of the $\mathrm{Ca}$ II IR triplet (Fig. 8). In the synthetic spectrum, the strength of the bluest of the two HV absorptions features is almost twice that of the red one. This is not the case in the observed feature and could be a 


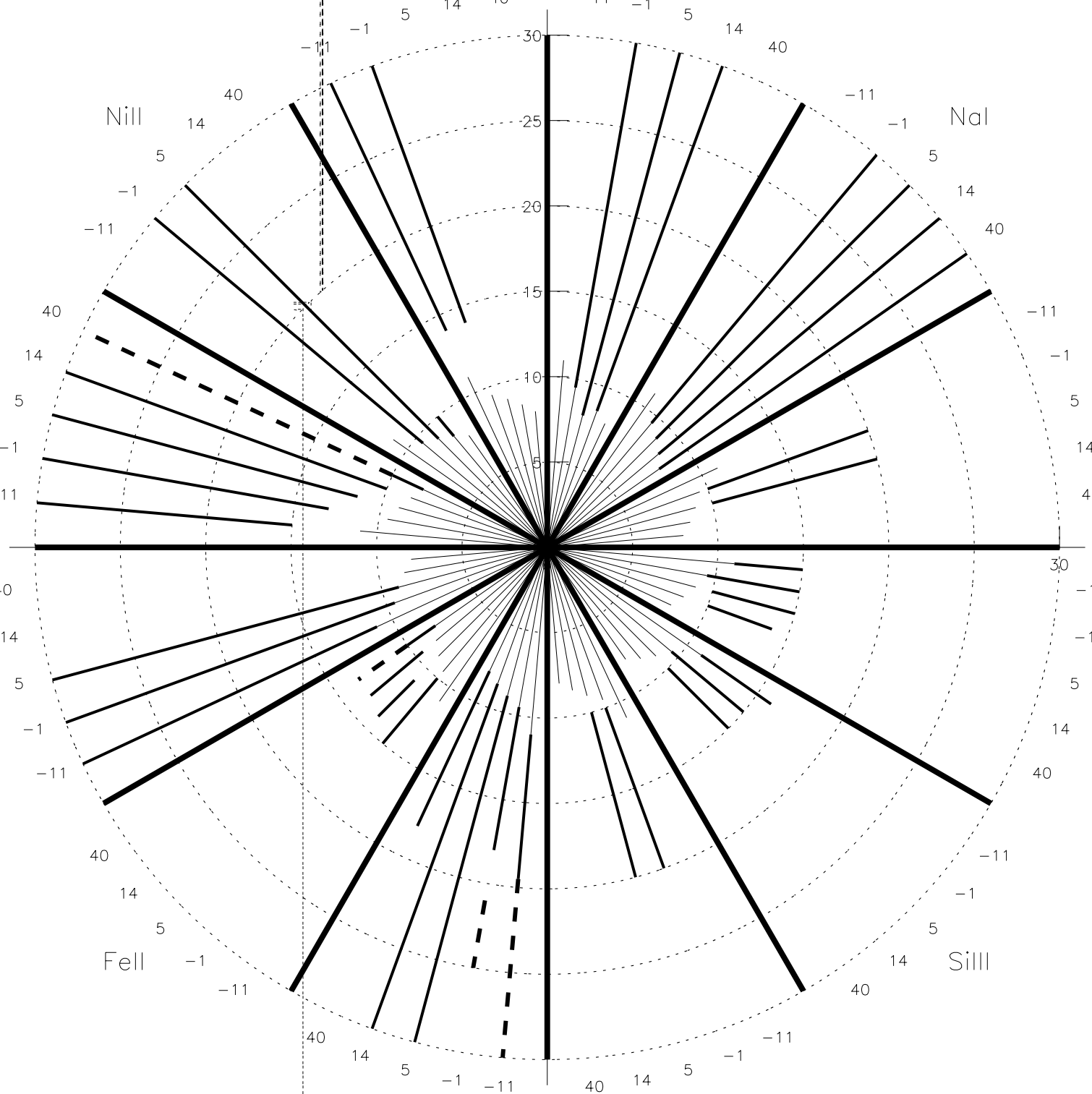

sign of asymmetry in the HV ejecta. To reproduce the correct flux level, a three-dimensional study of the geometry of the atmosphere (such as that computed for SN 2001el in Kasen et al. 2003) would be necessary. An optically thick HV clump viewed from a line of sight such that it just barely covers the photosphere can produce a weak IR triplet feature with two minima of equal depth (Kasen et al. 2003), such as seen in SN 1999aa.

Evidence for an $\mathrm{HV}$ component of $\mathrm{Ca}$ II also comes from the parameters in our synthetic spectra needed to model observed $\mathrm{Ca}$ II $\mathrm{H}$ and $\mathrm{K}$. To completely reproduce the broad profile of this line at day -11 and the blue minimum at day -1 the HV component is needed. When the temperature of the atmosphere drops, and the photosphere recedes into the inner layers, lowionization Fe-peak group lines become more important in the
$\mathrm{Ca} \mathrm{H}$ and $\mathrm{K}$ region. In this case, reproducing the line profile accurately becomes difficult. However, we could not match the $\mathrm{Ca} \mathrm{H}$ and $\mathrm{K}$ absorption shown at day -11 as well as at day -1 by any means other than an HV Ca II component. The evolution of this feature shows that the red component becomes stronger and that by 3 weeks after maximum it dominates the entire feature. This would be consistent with a decreasing optical depth of the HV Ca H and $\mathrm{K}$ as the atmosphere expands. This is consistent with our interpretation, namely, that the $\mathrm{HV} \mathrm{Ca}$ II component could be formed in a clump.

Recent spectropolarimetry measurements of SNe Ia (Howell et al. 2001; Wang et al. 2003; Kasen et al. 2003) have shown that, when present, the HV component of the Ca II IR triplet is usually polarized. The study of the polarization parameters can yield important information on the geometry 
of the explosion and thus can help in the development of threedimensional models.

\section{CONCLUSIONS}

We have presented new high signal-to-noise ratio optical spectroscopic data for SN 1999aa with good temporal coverage between -11 and +58 days with respect to $B$-band maximum light. The overall evolution of the spectral features suggests an object with characteristics common to both SN 1991T-like and Branch-normal supernovae.

By means of SYNOW synthetic spectra we have attempted to identify the absorption features in the spectra at days -11 , $-1,+5,+14$, and +40 with respect to $B$-band maximum light. Highlights of this modeling include the presence of $\mathrm{C}$ III at the photospheric velocity, possible $\mathrm{C}$ II or $\mathrm{H}$ at high-velocity, $\mathrm{Ca}$ II IR triplet at high-velocity, doubly ionized $\mathrm{Si}, \mathrm{Ni}$, and $\mathrm{Fe}$, confined IMEs and $\mathrm{Fe}$ II, and probable iron-peak core at $10,000 \mathrm{~km} \mathrm{~s}^{-1}$. A schematic view of the resulting composition (in the velocity regime) is presented in Figure 23 .

The line identification for the earliest spectrum shows the presence of doubly ionized $\mathrm{Si}, \mathrm{Fe}$, and $\mathrm{Ni}$, suggesting that the temperature in the outermost layer of SN 1999aa is higher than in normal SNe. After day +5 , doubly ionized elements no longer form visible absorption features, highlighting the cooling of the supernova atmosphere. The presence of IMEs above $14,000 \mathrm{~km} \mathrm{~s}^{-1}$ excludes the possibility that SN 1999aa was the result of a sub-Chandrasekhar mass explosion.

The broad absorption feature around $6150 \AA$ in the spectrum at day -11 can be matched comparably well by a weak component of Si II $\lambda 6355$ plus HV C II $\lambda 6580$ or $\mathrm{H} \alpha$. The contribution of either $\mathrm{C}$ II or $\mathrm{H} \alpha$ helps in reproducing the velocity time evolution of the absorption feature at $6150 \AA$ that otherwise (i.e., considering only Si II 26355 ) would remain unexplained. Definitive identification is not possible because of the lack of other absorption features produced either by $\mathrm{C}$ II or $\mathrm{H}$ at the input optical depths. However, both the possibilities impose constraints either on the nature of the progenitor system or the hydrodynamics of the explosion. A hydrogen component would imply that SN 1999aa is the result of a single degenerate WD explosion; a complete three-dimensional calculation would be necessary to model it. Alternatively, a C II component can be reproduced by a pure one-dimensional deflagration model, but would not be consistent with delayeddetonation models.

C III $\lambda 4648.8$ at photosphere velocity was able to reproduce the absorption around $4500 \AA$, and no good alternatives were found for that feature. If this identification is confirmed by detailed radiative transfer models, the use of three dimensional simulations of the explosion would be necessary to describe the presence of unburned material down to the inner layers of the WD atmosphere.

At day -11 and at day -1 , the profile of the absorption at $3800 \AA$ requires an $\mathrm{HV}$ component of $\mathrm{Ca}$ to be reproduced in addition to the photosphere component. The HV component at day -1 also reproduces the observed minima on the blue side of the usual Ca IR triplet. For an accurate description of this feature, multidimensional simulations would be required.
The spectral evolution of SN 1999aa in the photospheric phase shows that $\mathrm{Fe}$ II is confined below $15,000 \mathrm{~km} \mathrm{~s}^{-1}$. Intermediate mass elements populate a narrow velocity window above $10,000 \mathrm{~km} \mathrm{~s}^{-1}$. Similar evidence is found in other wellknown supernovae (SN 1991T, SN 1997br, and SN 2000cx) by studying the time evolution of the expansion velocity as computed from fits to the minima of Si II 26355 . The comparison with other SN 1991T-like objects suggests that the transition between IME to iron-peak dominant composition can occur at slightly different phases. Tuning of the deflagration to detonation transition density might reproduce this sequence.

The analysis of the spectrum of day +40 indicates that the iron-peak core limit should be set to $10000 \mathrm{~km} \mathrm{~s}^{-1}$, similar to that of SN 1991T (Fisher et al. 1999).

The origin of the differences between normal supernovae and SN 1991T-like or SN 1999aa-like objects depends on several factors. Our analysis of the optical spectra of SN 1999aa reveals that, among the present explosion models, none are able to reproduce each one of our findings. Higher temperature could account for some of the peculiarities (Nugent et al. 1995), but the evidence for high-velocity components and unburned material at all velocities probably requires the development of full NLTE three-dimensional explosion models. SN 1999aa was spectroscopically less extreme than other genuine SN 1991T-like SNe, suggesting that perhaps a single model could eventually explain both normals and SN 1991Tlike $\mathrm{SNe}$ as a continuous sequence.

We would like to thank Rollin Thomas for helpful comments and David Branch, Adam Fisher, and Rollin Thomas for providing the SYNOW code. We are grateful to the referee Mario Hamuy for useful suggestions. The research presented in this article made use of the SUSPECT ${ }^{32}$ Online Supernova Spectrum Archive and the atomic line list of Kurucz (1993). This work is based on observations made with the Nordic Optical Telescope, operated on the island of La Palma jointly by Denmark, Finland, Iceland, Norway, and Sweden, in the Spanish Observatorio del Roque de los Muchachos of the Instituto de Astrofisica de Canarias; the Apache Point Observatory 3.5 meter telescope, which is owned and operated by the Astrophysical Research Consortium; the Lick Observatory Shane $3.0 \mathrm{~m}$ telescope; the Cerro Tololo Inter-American Observatory $4 \mathrm{~m}$ Blanco telescope; the MDM Observatory $2.4 \mathrm{~m}$ Hiltner telescope. We thank the telescope allocation committees and the observatory staffs for their support for this extensive observing campaign. This work was supported in part by The Royal Swedish Academy of Sciences and by the Director, Office of Science, Office of High Energy and $\mathrm{Nu}-$ clear Physics, of the US Department of Energy under contract DE-AC03-76SF000098. A. M. acknowledges financial support from Fundação para a Ciência e Tecnologia (FCT), Portugal, through project PESO/P/PRO/15139/99; S. F. thanks the fellowship grant provided by FCT through project POCTI/ FNU/43749/2001. A. G. is a Royal Swedish Academy Research Fellow supported by a grant from the Knut and Alice Wallenberg Foundation.

\footnotetext{
${ }^{32}$ See http://www.nhn.ou.edu/ suspect.
}

\section{REFERENCES}

Aldering, G. 2000, in AIP Conf. Proc. 522, Cosmic Explosions: Tenth Astrophysics Conference, ed. S. S. Holt \& W. W. Zhang (New York: AIP), 75 Q6
Arnett, D. W. 1969, Ap\&SS, 5, 180

Baron, E., et al. 2000, ApJ, 545, 444

Baron, E., Hauschildt, P. H., Nugent, P., \& Branch, D. 1996, MNRAS, 283, 297 
Baron, E., Lentz, E. J., \& Hauschildt, P. H. 2003, ApJ, 588, L29

Bohlin, R. C., Colina, L., \& Finley, D. S. 1995, AJ, 110, 1316

Bohlin, R. C., Dickinson, M. E., \& Calzetti, D. 2001, AJ, 122, 2118

Branch, D. 2001a, in AIP Conf. Proc. 565, Young Suprnova Remnants (New York: AIP)

2001b, PASP, 113, 169

Branch, D., Baron, E., \& Jeffery, D. J. 2003a, in Supernovae and Gamma-Ray Bursts, ed. K. W. Weiler (Berlin: Springer)

Branch, D., Fisher, A., \& Nugent, P. 1993, AJ, 106, 2383

Branch, D., Garnavich, P., Matheson, T., Baron, E., Thomas, R. C., Hatano, K., Challis, P., Jha, S., et al. 2003b, AJ, 126, 1489

Branch, D., Lacy, C. H., McCall, M. L., Sutherland, P. G., Uomoto, A., Wheeler, J. C., \& Wills, B. J. 1983, ApJ, 270, 123

Cardelli, J. A., Clayton, G. C., \& Mathis, J. S. 1989, ApJ, 345, 245

Castor, J. I. 1970, MNRAS, 149, 111

Filippenko, A. V. 1997, ARA\&A, 35, 309

Q10 Filippenko, A. V., Li, W. D., \& Leonard, D. C. 1999, IAU Circ., 7108, 2

Filippenko, A. V., et al. 1992, ApJ, 384, L15

Fisher, A., Branch, D., Hatano, K., \& Baron, E. 1999, MNRAS, 304, 67

Fisher, A., Branch, D., Nugent, P., \& Baron, E. 1997, ApJ, 481, L89

Q11 Folatelli, G., et al. 2004, in preparation

Gamezo, V. N., Khokhlov, A. M., Oran, E. S., Chtchelkanova, A. Y., \& Rosenberg, R. O. 2003, Science, 299, 77

Garnavich, P. M., et al. 1998, ApJ, 493, L53

Q12 Garnavich, P. M., et al. 2001, ApJ, submitted (astro-ph/0105490)

Gibson, B. K., \& Stetson, P. B. 2001, ApJ, 547, L103

Gomez, G., Lopez, R., \& Sanchez, F. 1996, AJ, 112, 2094

Hamuy, M., et al. 2003, Nature, 424, 651

Hamuy, M., Suntzeff, N. B., Heathcote, S. R., Walker, A. R., Gigoux, P., \& Phillips, M. M. 1994, PASP, 106, 566

Hamuy, M., Walker, A. R., Suntzeff, N. B., Gigoux, P., Heathcote, S. R., \& Phillips, M. M. 1992, PASP, 104, 533

Hansen, C. J., \& Wheeler, J. C. 1969, Ap\&SS, 3, 464

Hatano, K., Branch, D., Fisher, A., Baron, E., \& Filippenko, A. V. 1999a, ApJ, 525,881

Hatano, K., Branch, D., Fisher, A., Millard, J., \& Baron, E. 1999b, ApJS, 121,233

Hatano, K., Branch, D., Qiu, Y. L., Baron, E., Thielemann, F.-K., \& Fisher, A. 2002, NewA, 7, 441

Hillebrandt, W., \& Niemeyer, J. C. 2000, ARA\&A, 38, 191

Hoeflich, P., Wheeler, J. C., \& Thielemann, F. K. 1998, ApJ, 495, 617

Horne, K. 1986, PASP, 98, 609

Howell, D. A., Höflich, P., Wang, L., \& Wheeler, J. C. 2001, ApJ, 556, 302

Iben, I., \& Tutukov, A. V. 1984, ApJS, 54, 335

Iwamoto, K., Brachwitz, F., Nomoto, K., Kishimoto, N., Umeda, H., Hix, W. R., \& Thielemann, F. 1999, ApJS, 125, 439

Jeffery, D. J., \& Branch, D. 1990, in Supernovae, Jerusalem Winter School for Theoretical Physics Vol. 6, ed. J. C. Wheeler, T. Piran, \& S. Weinberg (Singapore: World Scientific), 149

Jeffery, D. J., Leibundgut, B., Kirshner, R. P., Benetti, S., Branch, D., \& Sonneborn, G. 1992, ApJ, 397, 304

Jha, S. 2002, Ph.D. thesis, Harvard Univ.

Jha, S., et al. 1999, ApJS, 125, 73

Kasen, D., Wang, L., Howell, D. A., Wheeler, J. C., Höflich, P., Baade, D., Baron, E., \& Hauschildt, P. H. 2003, ApJ, 593, 788

Khokhlov, A. M. 1991, A\&A, 245, 114

Q13 . 2000, ApJ, submitted (astro-ph/0008463)

Kirshner, R. P., et al. 1993, ApJ, 415, 589

Knop, R. A., et al. 2003, ApJ, 598, 102

Krisciunas, K., Hastings, N. C., Loomis, K., McMillan, R., Rest, A., Riess, A. G., \& Stubbs, C. 2000, ApJ, 539, 658

Kurucz, R. L. 1993, Kurucz CD-ROM 1, Atomic Data for Opacity Calculations (Cambridge: SAO)

Leibundgut, B. 2000, A\&A Rev., 10, 179

Leibundgut, B., Kirshner, R. P., Filippenko, A. V., Shields, J. C., Foltz, C. B., Phillips, M. M., \& Sonneborn, G. 1991, ApJ, 371, L23

Lentz, E. J., Baron, E., Branch, D., \& Hauschildt, P. H. 2001, ApJ, 547,402
Lentz, E. J., Baron, E., Branch, D., Hauschildt, P. H., \& Nugent, P. E. 2000, ApJ, 530, 966

Lentz, E. J., Baron, E., Hauschildt, P. H., \& Branch, D. 2002, ApJ, 580, 374

Li, W., et al. 2001a, PASP, 113, 1178

Li, W., Filippenko, A. V., Treffers, R. R., Riess, A. G., Hu, J., \& Qiu, Y. 2001b, ApJ, 546, 734

Li, W. D., et al. 1999, AJ, 117, 2709

Livio, M. 2001, in STScI Symp. 13, Supernovae and Gamma-Ray Bursts, ed. M. Livio, N. Panagia, \& K. Sahu (Cambridge: Cambridge Univ. Press), 334 Livio, M., \& Riess, A. G. 2003, ApJ, 594, L93

Livne, E. 1990, ApJ, 354, L53

Livne, E., \& Arnett, D. 1995, ApJ, 452, 62

Livne, E., \& Glasner, A. S. 1991, ApJ, 370, 272

Lucy, L. B. 1991, ApJ, 383, 308

Marietta, E., Burrows, A., \& Fryxell, B. 2000, ApJS, 128, 615

Marquardt, D. 1963, SIAM J. Appl. Math., 2, 2

Massey, P., \& Gronwall, C. 1990, ApJ, 358, 344

Mazzali, P. A. 2001, MNRAS, 321, 341

Mazzali, P. A., Cappellaro, E., Danziger, I. J., Turatto, M., \& Benetti, S. 1998, ApJ, 499, L49

Mazzali, P. A., Danziger, I. J., \& Turatto, M. 1995, A\&A, 297, 509

Mazzali, P. A., Lucy, L. B., Danziger, I. J., Gouiffes, C., Cappellaro, E., \& Turatto, M. 1993, A\&A, 269, 423

Nakano, S., Kushida, R., \& Kushida, Y. 1999, IAU Circ., 7109, 4

Nomoto, K. 1982, ApJ, 257, 780

Nomoto, K., Sugimoto, D., \& Neo, S. 1976, Ap\&SS, 39, L37

Nomoto, K., Thielemann, F.-K., \& Yokoi, K. 1984, ApJ, 286, 644

Nugent, P., Aldering, G., \& The Nearby Campaign. 2000, in Poster Papers, STScI Symp., Supernovae and Gamma-Ray Bursts, ed. M. Livio, N. Panagia, \& K. Sahu (Baltimore: STScI), 47

Nugent, P., Baron, E., Branch, D., Fisher, A., \& Hauschildt, P. H. 1997, ApJ, 485,812

Nugent, P., Phillips, M., Baron, E., Branch, D., \& Hauschildt, P. 1995, ApJ, 455, L147

Oke, J. B. 1990, AJ, 99, 1621

Paczynski, B. 1985, Cataclysmic Variables and Low-Mass X-Ray Binaries (Dordrecht: Kluwer), 1

Patat, F., Benetti, S., Cappellaro, E., Danziger, I. J., della Valle, M., Mazzali, P. A., \& Turatto, M. 1996, MNRAS, 278, 111

Perlmutter, S., et al. 1998, Nature, 391, 51

. 1999, ApJ, 517, 565

Phillips, M. M. 1993, ApJ, 413, L105

Phillips, M. M., Wells, L. A., Suntzeff, N. B., Hamuy, M., Leibundgut, B., Kirshner, R. P., \& Foltz, C. B. 1992, AJ, 103, 1632

Qiao, Q. Y., Wei, J. Y., Qiu, Y. L., \& Hu, J. Y. 1999, IAU Circ., 7109, 3

Riess, A. G., et al. 1998, AJ, 116, 1009

Ruiz-Lapuente, P., Cappellaro, E., Turatto, M., Gouiffes, C., Danziger, I. J., della Valle, M., \& Lucy, L. B. 1992, ApJ, 387, L33

Saha, A., Sandage, A., Thim, F., Labhardt, L., Tammann, G. A., Christensen, J., Panagia, N., \& Macchetto, F. D. 2001, ApJ, 551, 973

Salvo, M. E., Cappellaro, E., Mazzali, P. A., Benetti, S., Danziger, I. J., Patat, F., \& Turatto, M. 2001, MNRAS, 321, 254

Schlegel, D. J., Finkbeiner, D. P., \& Davis, M. 1998, ApJ, 500, 525

Schmidt, B. P., et al. 1998, ApJ, 507, 46

Sobolev, V. V. 1960, Moving Envelopes of Stars (Cambridge: Harvard Univ. Press)

Stone, R. P. S. 1996, ApJS, 107, 423

Thomas, R. C., Branch, D., Baron, E., Nomoto, K., Li, W., \& Filippenko, A. V. 2003, ApJ, 601, 1019

Thomas, R. C., Kasen, D., Branch, D., \& Baron, E. 2002, ApJ, 567, 1037

Tonry, J. L., et al. 2003, ApJ, 594, 1

Turnshek, D. A., Bohlin, R. C., Williamson, R. L., Lupie, O. L., Koornneef, J., \& Morgan, D. H. 1990, AJ, 99, 1243

Wang, L., et al. 2003, ApJ, 591, 1110

Wang, L., Wheeler, C. J., \& Hoeflich, P. 1996, BAAS, 28, 1332

Whelan, J., \& Iben, I. J. 1973, ApJ, 186, 1007

Woosley, S. E., \& Weaver, T. A. 1994, ApJ, 423, 371

Yamaoka, H., Nomoto, K., Shigeyama, T., \& Thielemann, F. 1992, ApJ, 393, L55 


\section{AUTHOR QUERIES}

Q1 Au: Is there evidence of $\mathrm{C}$ and $\mathrm{O}$ lines at low velocities, as well as clumps of burned material? If so the sentence should probably be changed. Perhaps to "tends to confirm"?

Q2 Au: The phrase "was subtracted to" has been changed to "was subtracted from." OK?

Q3 Au: In this sentence do you mean " the processes that affect the continum emission," Or the process that shape the continum emission"?

Q4 Au: The phrase "the absorption on the red of Si III" has been changed to "the absorption to the red of Si III." Is this OK or would you prefer "in the red of Si III"?

Q5 Au: The "Synthetic Spectrum" sections required section numbers. It seemed appropriate to make them subsections rather than first-level sections. The text references to the first-level sections should still be correct. Is this OK?

Q6 Au: For Armstrong \& Schwartz the number of the IAU Circular has been added. Is this correct?

Q7 Au: Blinnikov \& Sorokina, has been updated. Is this correct?

Q8 Au: Branch 2000 has been updated and changed to 2001a, while the next entry is now Branch 2001b. Is this OK?

Q9 Au: Branch, Baron, \& Jeffery 2001 has been updated to Branch, Baron, \& Jeffery 2003a. Is this OK?

Q10 Au: The IAU Circular number has been added to Filippenko, Li, \& Leonard 1999. OK?

Q11 Au: Do you have an update for Folatelli et al. 2004?

Q12 Au: Do you have an update for Garnavich et al. 2001?

Q13 Au: Do you have an update for Khokhlov 2000?

Q14 Au: The IAU Circ. number has been added to Qiao et al. 1999. Is this correct?

Q15 Au: Thomas, Branch, Baron, Nomoto, Li, \& Filippennko 2003 has been updated. The entry for Thomas, R. C., et al. 2003, astroph/0302260 has been removed, and all citations for it directed to the former, since they refer to the same paper. OK? 